\title{
Probing adsorbent heterogeneity using Toth isotherms
}

\author{
K. Vasanth Kumar,,${ }^{1,2 *}$ S. Gadipelli, ${ }^{3,4}$ Christopher A. Howard,${ }^{5}$ W. Kwapinski, ${ }^{1}$ D. J. L. Brett ${ }^{4}$ \\ ${ }^{1}$ Department of Chemical Sciences, University of Limerick, Limerick, Ireland. \\ ${ }^{2}$ Synthesis and Solid State Pharmaceutical Centre, Bernal Institute, University of Limerick, Limerick, Ireland. \\ ${ }^{3}$ College of Physics, Sichuan University, Chengdu, 610064, China. \\ ${ }^{4}$ Electrochemical Innovation Lab, Department of Chemical Engineering, UCL, London, United Kingdom. \\ ${ }^{5}$ Department of Physics \& Astronomy, University College London, London WC1E 6BT, \\ United Kingdom.
}

*Correspondence: Vasanth.kannuchamy@ul.ie

\begin{abstract}
The binding affinity distribution is a fingerprint of the adsorbent heterogeneity. In chromatographic separations, the affinity distribution determines the molecular recognition capability of the stationary phases. In gas storage and separation applications, the affinity distribution decides the selectivity towards a target molecule. The end application of almost every molecular recognizing material like molecularly imprinted polymers (MIPs) critically depend on their affinity distribution. In this work, we develop two simple analytical expressions by coupling a numerical technique with the widely used Toth isotherms. These models are universally applicable and can accurately characterize different classes of industrially-important adsorbents that include metal organic frameworks, carbon allotropes and chromatographic stationary phases for their selectivity, binding affinity and adsorption site heterogeneity. The models require only the Toth isotherm parameters to characterize both homogeneous or heterogeneous adsorbents.
\end{abstract}

Keywords: adsorption, porous materials, heterogeneity, adsorption site, binding energy, binding energy distribution, Toth isotherm, discrete model, continuous distribution model.

\section{Introduction}

Materials with porosity levels, ranging from a few percent to as high as 90 percent, with specific surface areas ranging from a few $\mathrm{m}^{2} / \mathrm{g}$ to as high as $7000 \mathrm{~m}^{2} / \mathrm{g}$ and different surface chemistries, have found a broad range of applications in the area of adsorption science and catalysis. These materials can act as adsorbents used to separate targeted molecules from a bulk fluid (gas or liquid) or to separate a specific molecular species from a mixture of gases. Recently, porous materials, especially those with high surface area, are investigated for: the storage of fluids such as $\mathrm{CH}_{4}$ and $\mathrm{H}_{2}$; to capture $\mathrm{CO}_{2}$ from flue gas, for air filtration; and to capture of toxic gases and volatile organic vapours. ${ }^{1,2,11-14,3-10}$ For instance, carbon nanotubes and metal-organic frameworks (MOFs) can potentially 'recognize' (i.e. specifically interact with) small molecules and thus be used for gas sensing. ${ }^{15-18}$ In the area of liquid-phase adsorption, porous materials are used to remove targeted pollutants from wastewater. ${ }^{19-21}$ For example, molecularly-imprinted polymers that can exclusively recognize a specific molecule, can be used as gas sensors and chromatographic stationary phases. ${ }^{22-24}$ All of these technologies exploit the ability of the porous material adsorbent to selectively concentrate specific molecules on their surface or within their pore volume from a large volume of bulk fluid.

Adsorption depends on the concentration of the target molecule in solution and gas pressure in the bulk phase. The amount adsorbed also depends on the properties of the adsorbent, including surface area, pore-volume, concentration of functional groups and pore size distribution. The common practice in research laboratories is to determine the effect of each of these parameters individually on the amount adsorbed. Alternatively, the combined effect of these parameters is captured by modelling the adsorption site heterogeneity often simply referred to as the

Page | 1 
'heterogeneity' of the adsorbent. ${ }^{25}$ The adsorption site heterogeneity can be estimated by modelling the number of adsorption sites in the solid adsorbent and their types. ${ }^{25-28}$ Both of these parameters ultimately underpin the capability of an adsorbent to bind a specific molecule from a bulk gas or from a solution that contains both solute and solvent at a particular temperature and concentration or pressure.

Adsorption varies with pressure or concentration due to the presence of different types of active sites with varied adsorption strengths, i.e., each type having a different binding affinity on the adsorbent surface. If the adsorbent contains more than one type of adsorption site, then the material is heterogeneous. The simplest heterogeneous adsorbent is the one that contains only two types of binding sites, distinguished by their binding affinity (sites with higher affinity and sites with lower affinity). The higher binding affinity sites can host target molecules in gas phase at relatively lower pressures, whereas sites with lower binding affinity can host the guest species only at higher pressures or concentrations. Binding site heterogeneity is a common property of many materials like molecularly imprinted polymers, polymers with intrinsic porosity (PIMs), composite structures and the most widely used activated carbons.

In some cases, heterogeneity in some cases can determine the applications of some of the materials discussed above. For instance, the molecular recognition efficacy of the molecularly imprinted polymers is limited by the level of heterogeneity. ${ }^{27,29,30}$ Gas storage materials are often characterised for their pore and surface chemical properties using $\mathrm{N}_{2}$, Ar and $\mathrm{CO}_{2}$ adsorption isotherms and spectroscopic techniques, irrespective of the end applications. ${ }^{31}$ The pore properties and surface chemical properties are later correlated with the adsorption properties of the materials for a specific guest molecule that may be indifferent to the properties of $\mathrm{N}_{2}$ and $\mathrm{CO}_{2}$. Alternatively, the adsorbents can be characterised in the light of affinity spectra obtained from the adsorption isotherms measured with the actual probe molecules of interest, rather than using $\mathrm{N}_{2}$ and $\mathrm{CO}_{2}$ adsorption isotherms. Affinity spectra reflect the characteristic heterogeneity of the adsorbents and it correlates the relative amounts of the different types of binding sites.Affinity spectra are also specific to adsorbate molecules and the adsorption conditions and are sensitive to interactions at the interface between the adsorbate molecule and the adsorbent surface. This means, theoretically the performance of different adsorbents can be compared in terms of the number and types of adsorption sites available on the surface and their specific binding affinity for different adsorbates. For instance, adsorbent for $\mathrm{CO}_{2}$ capture, can be tested for $\mathrm{CH}_{4}, \mathrm{~N}_{2}$ and $\mathrm{H}_{2}$ to elucidate the heterogeneity or its role. Such a plot can be used to screen adsorbents for the separation of mixture molecules. Additionally, comparison between different classes of porous materials can be performed in terms of adsorbent heteogeneity. It is also possible to compare two different materials and check the number of sites under each type of materials with a certain binding affinity. This feature will help to characterise the adsorption type/mechanism, and identify special cases for example the same material being homogeneous and heterogeneous for the same target molecule as a function of temperature. ${ }^{32-34}$ Heterogeneity can also serve as a guide while designing or characterising the chromatographic column materials with the retention behaviour of different probe molecules.

Heterogeneity is often characterised using discrete models like a bi-Langmuir or in general multi-site Langmuir isotherms, which assumes there exists some discrete number of different types of adsorption sites. ${ }^{26,28,35,36}$ These discrete models often produce ambiguous results and in many cases, it is difficult to compare the heterogeneity of different adsorbents since the number of adsorption sites and their binding energy vary. Thus, an alternative method that can produce unambiguous information about the types of adsorption sites, number of sites under each class and their binding affinity for a specific target molecule would be hugely beneficial. Continuous distribution functions are considered to be an improvement upon the discrete binding models. ${ }^{37,28}$ In the literature, there have been many attempts to develop such mathematical functions using different approaches. Our recent work has reviewed the different methods used to develop continuous distribution functions. ${ }^{25}$ Continuous distribution functions are mathematical functions that allow the generation of an affinity spectrum, which is a plot of the binding affinity (also called the association constant) against the number of adsorption sites with that binding affinity (this is discussed in detail in the later sections). Given that most adsorbents are intrinsically heterogeneous, and considering the 
importance of this adsorbent property, in this work we propose two simple continuous distribution functions by coupling the the two versions of the Toth isotherm and a numerical technique.

The numerical technique used in this study was was originally developed by Ninomiya and Ferry ${ }^{38}$ and was first used by Hunston to generate affinity spectra ${ }^{28}$. Although this method does not require any initial assumptions on the shape of the distribution spectra, it requires a large number of experimental equilibrium data points and timeconsuming calculations. These requirements limit the usefulness of this technique and make them system-specific rather than universal. However, the numerical technique can be fully exploited if we assume the binding affinity spectra is of a specific form. The affinity distribution can be assumed to follow an exponential form based on the Freundlich isotherm. ${ }^{27}$ However, the Freundlich isotherm is not universal and can only be applied within certain concentration or pressure limits nor can it capture the saturation behaviour or model homogeneous adsorbents. ${ }^{30}$ Earlier work used a Langmuir-Freundlich isotherm to capture, both homogeneous and heterogeneous distribution of binding site affinities. ${ }^{29}$

In this work, we selected two versions of the Toth isotherm to model both a homogeneous and a heterogeneous distribution of adsorption site affinities (see section 2) ${ }^{39,40}$ Both of these expressions assume a quasi-Gaussian distribution of binding site affinities. In fact, the Toth isotherm is one of the most widely used isotherms to model both liquid and gas phase adsorption equilibrium data. We then used the proposed mathematical function to characterise the heterogeneity of a number of industrially important adsorbents from different classes of materials that include fourteen different molecularly imprinted polymers, two benchmark (for methane adsorption) MOFs and four benchmark (for methane/ $\mathrm{CO}_{2}$ adsorption) carbon-based materials, three silica-based adsorbents materials in chromatographic columns. We also studied a system that involves adsorption of multiple-solutes from bulk gas or solution. These materials/systems include those widely used in the area of wastewater treatment, gas storage/separations, gas/liquid sensing, chromatographic stationary columns, separation and purification of drugs and biological compounds, molecular recognition and separation of racemic mixtures. These systems differ from each other in terms of factors including pore properties like surface area and pore volume, surface chemistry, adsorbate/adsorbent interfacial regions, adsorbate phase (liquid or gas), pore structure, crystallinity, different end applications. These systems were carefully selected to demonstrate the universality of the proposed mathematical functions for capturing both the homogeneous and heterogeneous distribution of binding site affinities in different classes of materials for both gas and liquid phase adsorption systems. Moreover, we demonstrate how the proposed model can elucidate the commonly encountered issue in the area of adsorptive wastewater treatment of the effect of pollutants or a second solute that competes with the targeted molecules. Finally, the proposed model was used to explain the experimental paradox of the higher retention of phenol than caffeine in silica columns. Throughout the manuscript, the heterogeneity is used to expose what type of binding sites participate in the adsorption and how they respond when exposed to target molecules. It is also emphasized how heterogeneity alone allows comparisons to be made between the performance of different adsorbents without requiring information about the pore, structural or surface properties. Finally, a concise review is provided of the continuous distribution models and comparison made between the proposed models and existing models in the literature.

\section{Theory}

\subsection{A model homogeneous and heterogeneous carbon surface at the atomic level and the concept of binding affinity}

Figure 1a depicts a model of a carbon surface that usually bounds a pore and the different types of binding sites, in the forms of Fe-doped carbon surfaces, $\mathrm{N}$ heteroatoms, hydroxyl and carboxylic groups, that can be expected to exist in a activated carbon. A carbon (Figure 1b) composed by this type of surface can be taken as a typical example of a heterogeneous adsorbent. On the other hand, homogeneous adsorbents are simple and contain only one type of adsorption site. An ideal graphene sheet or a simple slit-shaped carbon pores can be taken as examples to represent homogeneous surface and homogeneous adsorbent, respectively. Another example of homogeneous adsorbent is a MIP. In Figure 1c, we show a

Page | 3 
computer generated model of a MIP that contains binding pockets or sites that can fit norflaxicin. As this material contains only one type of binding pockets and can be classified as homogeneous adsorbent.

The different types of adsorption sites shown in Figure 1a-b are only representative; in real carbon structures, the number and the type of adsorption sites cannot be simply counted and it is a theoretical challenge to obtain the actual number on the types of adsorption sites using analytical techniques. In addition to surface functionalities, heterogeneity is also influenced by atomic site vacancies, the location of functional groups and how they are distributed on the carbon surface. ${ }^{41-43}$ The combination of all of these factors makes the overall distribution impossible to capture using any analytical techniques and thus it is essential to rely on theoretical methods that allows to get approximate information about the types of adsorption sites available on such complex adsorbent surfaces and their binding affinity. Although such theoretical methods cannot predict which types of sites (e.g., atomic defects, functionalities) posses a specific affinity, they can expose the number of different types of adsorption sites.
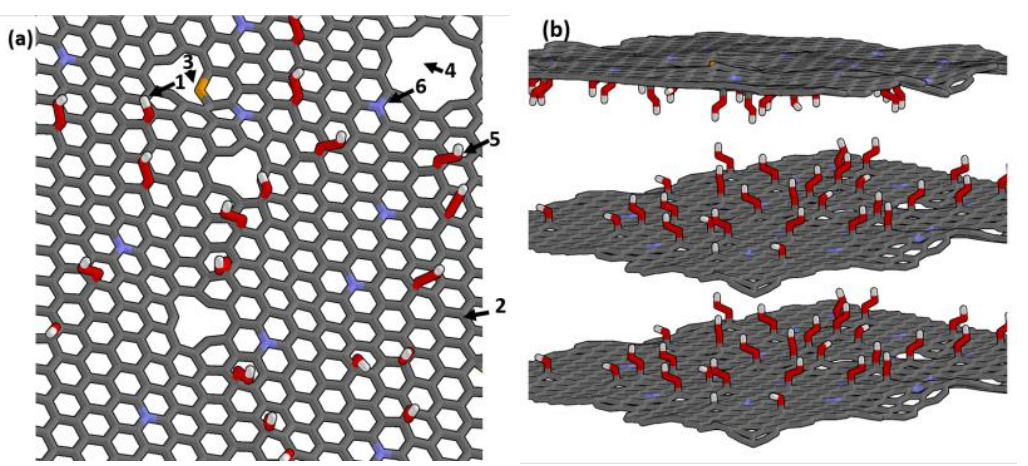

(c)

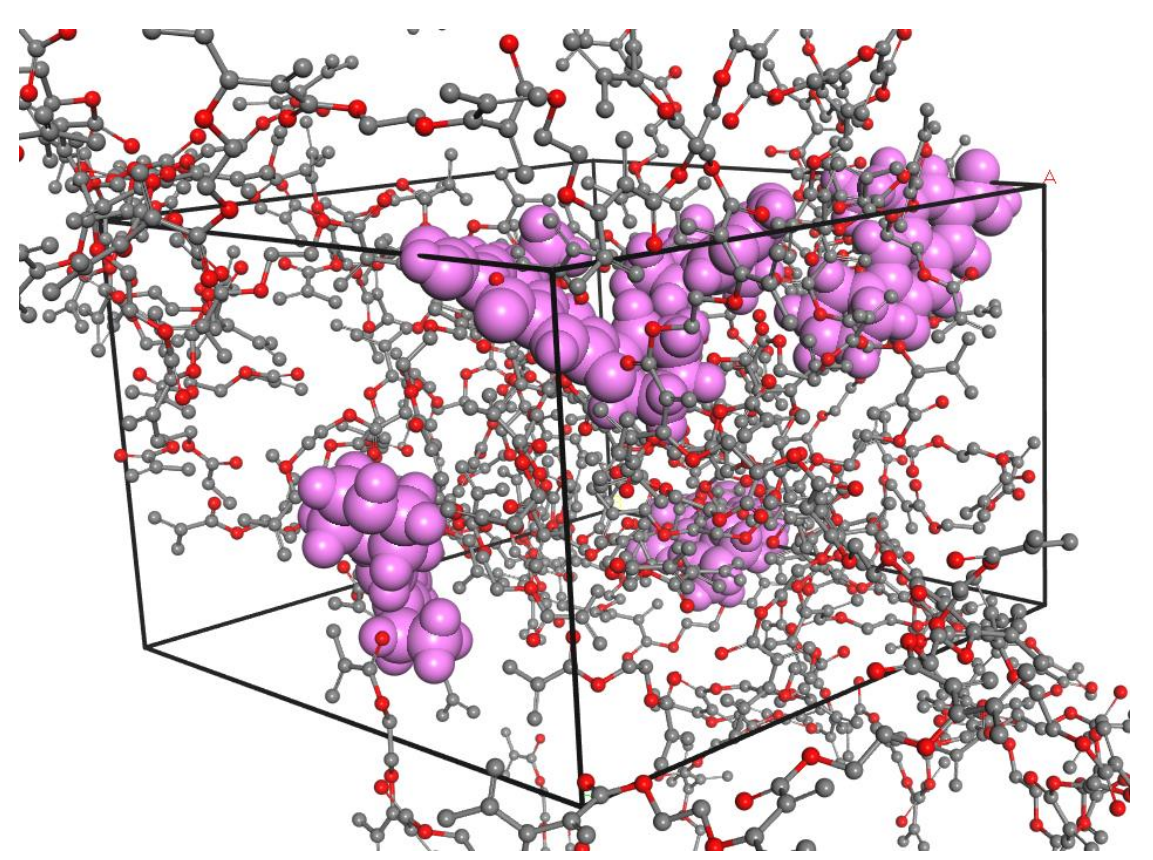

Figure 1: (a) A model pore surface of a Fe and N-doped graphitic carbon prepared via chemical/physical activation methods. It is possible to identify six different types of adsorption site on the pore surface: (1) hydroxyl groups, (2) pristine graphitic carbon surface, (3) Fe atoms, (4) atomic site vacancies (which can sometimes create a surface curvature), (5) carboxyl groups, (6) $\mathrm{N}$ atoms; (b) A prototype of an $\mathrm{Fe}, \mathrm{N}$ doped activated carbon with surfaces enriched with oxygenated (hydroxyl and carboxylic) groups that can be approximate functionalised slit-shaped pores observed in activated carbons, and (c) a computer generated model of a molecularly imprinted polymer loaded with norflaxacin (shown as mauve vDW spheres).

Page | 4 
Though the stucuture is made up of an amorphous polymeric matrix, it contains only type of binding site that can recognize only norflaxacin molecules making this material homogneous.

\subsection{Homogeneous versus Heterogeneous adsorbents}

One way to understand adsorption site heterogeneity is to assume that several types of adsorption sites exist, spread over a two-dimensional surface. Figure 2a-c shows a two-dimensional representation of three different types of adsorbent surfaces: (i) homogeneous adsorbent with a binding affinity of $K_{1}$, (ii) simplest heterogeneous surface: an adsorbent surface that contains only two types of adsorption sites; this can be adsorption sites with a higher binding affinity $\left(K_{1}\right)$ and lower binding affinity $\left(K_{2}\right)$, (iii) heterogeneous and a complex adsorbent surface that contains more than two types of adsorption sites. The model surface shown in Figure 2c contains six different types of adsorption sites with different levels of binding affinity ranging from $K_{l}$ to $K_{6}$ (where $K_{1}>K_{2}>K_{3}>K_{4}>K_{5}>K_{6}$ ).

The affinity distribution of the three different adsorbent surfaces (top panel) is shown in bottom panels of Fig. 2.

Most of the commonly used adsorbents are heterogeneous and the binding affinities will not be as simplistic as the one shown in Figure $2 b$ or $2 c$. The adsorbent surfaces might effectively contain a large number and types of adsorption sites which may be difficult to treat by simply assuming that there exist some discrete types of binding sites on an adsorbent surface. In many cases, the binding affinity can be exponentially distributed for a highly heterogeneous surface and in some cases, the adsorption site binding affinities will be uniformly distributed. If the distribution is uniform, heterogeneity can be characterised based on the width of the distribution.

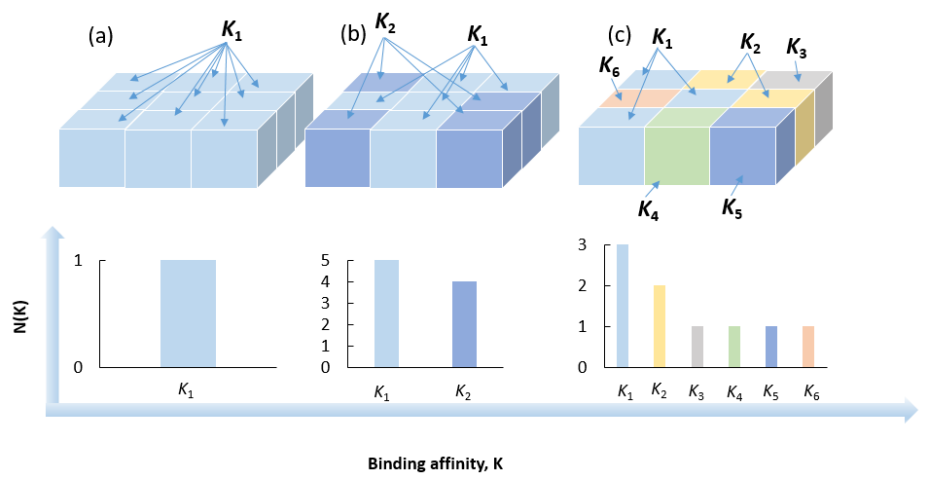

Fig. 2 Two-dimensional representation of (a) homogenous surface, (b) simplest heterogeneous surface and (c) a slightly complex heterogeneous surface. Bottom panel: affinity distribution plot, $N\left(K_{\mathrm{i}}\right)$ versus $K_{\mathrm{i}}$ based on the number of adsorption sites (where $i$ refers to the number of type of adsorption sites; if $i=6$, then there are six different types of binding sites that differ by binding affinity).

\section{Determination of the number and type of adsorption sites}

The number of adsorption sites and their types are not known a priori but can be calculated using two different techniques. In the first technique, the information is obtained from a Scatchard plot based on a simple Langmuir isotherm (see the theory below). This method requires some user assumptions about the number of different types of adsorption sites. In the second method, the number of adsorption sites and their types will be determined without requiring any prior assumptions using a simple numerical technique and a theoretical isotherm.

\subsubsection{Method 1: Scatchard plot technique using a discrete distribution model}

In 1918, Irving Langmuir proposed a mathematical expression for isotherm to describe the adsorption of gases on the plane surfaces of glass, mica and platinum. ${ }^{44}$ According to Langmuir, the amount adsorbed, $q\left(\mathrm{mmol} \mathrm{g}^{-1}\right)$ at equilibrium conditions can be related to the equilibrium gas pressure, $p$ (bar) of the bulk fluid by the relation

$q=\frac{N K p}{1+K p}$

Page | 5 
where $N$ is the maximum number of adsorption sites available on the adsorbent surface and is usually expressed in terms of

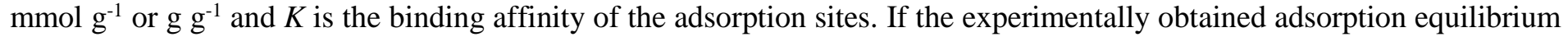
data follows Eq (1), then only one type of adsorption site exits on the adsorbent and only one adsorbed layer on the adsorbent surface. In other words, adsorption will increase with pressure until the entire adsorbent surface is covered by a layer of adsorbed molecules. Typically, the isotherm parameters are obtained from the linear expression of the Langmuir isotherm given by ${ }^{26,44}$

$\frac{p}{q}=\frac{1}{N K}+\frac{p}{N}$

The isotherm constants, $\mathrm{N}$ and $\mathrm{K}$ can be calculated from the plot of $p / q$ versus $p$ using linear regression analysis. For the case of liquid-phase adsorption, the variables $p$ and $q$ will be replaced by the concentration in the bulk solution, $C_{\mathrm{e}}$ and the amount adsorbed at equilibrium, $q_{\mathrm{e}}$, respectively. Here it is worth noting that the best-fit of the experimental equilibrium data in Eq (2) points to the fact that, irrespective of the different adsorption behaviour, there exists only one type of adsorption site on these adsorbents. Most of the adsorbents prepared in the laboratory are heterogeneous and thus the expression in Eq (2) cannot capture the adsorbent heterogeneity.

To deal with heterogeneous surfaces, a multi-site Langmuir isotherm can be used to determine the number and types of adsorbent sites. ${ }^{26,28,37}$

$q=\sum_{i=1}^{m} \frac{N_{i} K_{i} p}{1+K_{i} p}$

Where, $\boldsymbol{N}_{\boldsymbol{i}}$ is the number of adsorption sites with the binding affinity $K_{\mathrm{i}}$ for each of the $m$ classes of binding site. According to the above expression, the total number of adsorption sites, $N_{\mathrm{o}}$, can be defined by the expression

$N_{o}=\sum_{i=1}^{m} N_{i}$

$N_{\mathrm{o}}$ is just the sum of all the 'discrete' number of binding sites available on the adsorbent and this is not a function of $K_{\mathrm{i}}$. This is a unique parameter and it includes the total number of binding sites on the surface adsorbent.

Eq (3) was used by Scatchard ${ }^{35}$ to characterise protein-ligand interactions where an adsorbent surface can be assumed to contain different sub-microscopic patches or regions with uniform construction, and each of these patches is considered a homogeneous surface. If the adsorbent contains $m$ number of these sub-microscopic surface patches, then the adsorption on each of these patches can be represented by the Langmuir isotherm and the sum of the adsorption in all of those individual patches will be equal to the total adsorption. If the adsorbent contains only two types of adsorption sites or two types of surface patches at the sub-microscopic level, high affinity and low affinity binding sites, and if the adsorption on each of these behaves differently and the adsorption follows Langmuir model, then the whole process can be modelled using a biLangmuir isotherm:

$q=\frac{N_{1} K_{1} p}{1+K_{1} p}+\frac{N_{2} K_{2} p}{1+K_{2} p}$

where $K_{1}$ is usually much larger than $K_{2}$ and $N_{2}$ is much smaller than $N_{1}$.

Similarly, if an adsorbent contains three or four types of adsorption sites, then a tri-Langmuir and tetra-Langmuir isotherm can be used to determine the affinity parameters. If the adsorbent contains a greater number of adsorption sites, then the Langmuir isotherm can be extended accordingly and the affinity parameters can be determined.

Though it could be inferred that a bi-Langmuir, tri-Langmuir or the higher extensions of Langmuir isotherm can be used to determine the affinity parameters and to get an idea about the heterogeneity of adsorbents, there is no straightforward method 
to determine the types of adsorbent sites available on an adsorbent surface. To reveal the natural heterogeneity of the adsorbent or to determine the number of types of adsorption sites, it is essential to rewrite Eq (1) as follows

$\frac{q}{p}=N K-K q$

Eq (6) was originally proposed by Scatchard to estimate the affinity parameters involved during the attraction of small molecules and ions by proteins. ${ }^{35}$ This expression is applicable for both liquid and gas phase adsorption. For the case of liquid-phase adsorption, the affinity parameters will be determined from the plot of $q_{\mathrm{e}} / C_{\mathrm{e}}$ versus $q_{\mathrm{e}}$. According to Eq (6), it is possible to plot the experimental equilibrium data in the form of $q / p$ versus $q$. A linear trend-line in the plot of $q / p$ versus $q$ means the adsorbates are adsorbed onto only one type of adsorption sites (see the red trend-line in Figure 3; it shows the equilibirium data for two different adsorbents) and in this case, the binding affinity can be determined from the slope ( $K=-$ slope) and the $x$-intercept will be equal to the number of adsorption sites.

If the adsorbent contains more than one type of adsorption site, then the plot of $q / p$ versus $q$ will not be a straight line but a curve as per binding site heterogeneity. It is easy to assume that at least two classes of binding sites exist and therefore to draw at least two tangents on the curve (e.g. blue tangents in Figure 3). In this case, the experimental adsorption isotherm can follow the bi-Langmuir isotherm. The slope and intercept of each of the tangent can determine the number of adsorption sites and their binding energy (Eq (6)). Determining the affinity parameters from the $q / p$ versus $q$ plot is also called the graphical or limiting slope method; this plot allows two (or more) separate trend-lines to be drawn and thus yield two separate sets of binding parameters $\left(N_{1}, K_{1}\right.$ and $\left.N_{2}, K_{2}\right)$ for the two classes of binding sites.

In Figure 3, the steeper blue-line measures the high-affinity sites and the flatter blue-line measures the low-affinity sites. Identifying the different types of adsorption sites from the Scatchard expression is called a Scatchard analysis. The expressions that include the simple Langmuir isotherm, bi-Langmuir or in general multi-site Langmuir isotherm used in this method are called discrete distribution models as they simplify the distribution of adsorbent sites that may exist on the adsorbent surface into a finite number of different types of sites; each type of site having a different binding affinity. ${ }^{45}$

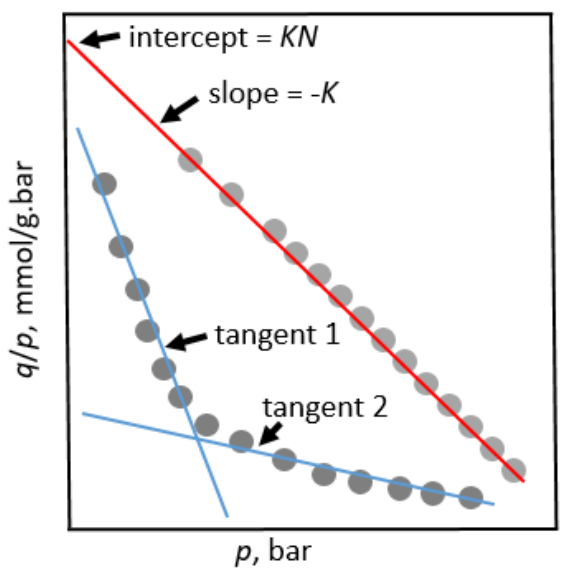

Figure 3: Equilibrium data plotted on a Scatchard plot for a homogeneous adsorbent that contains only one type of binding sites (red trend-line) and an adsorbent contains at least two different types of binding sites (two blue tangents) confirms the existence of two different types of binding sites.

This method has a major drawback as it groups the different type of binding sites into a finite number of categories and requires preliminary, assumptions on the number of tangents to be drawn on a Scatchard plot. The accuracy of this method also depends on the number of data points of experimental data. This method is ambiguous as it requires subjective user assumptions on which points to include in which subset. This technique will become even more complex with higher-order tri- and tetra-Langmuir models. These limitations can be eliminated by a non-linear regression technique (see Section 2.3 and 3.4).

\subsubsection{Method 2: Numerical technique using a continuous distribution (Toth isotherm) model.}


Continuous distribution functions assume a continuous distribution of binding constants and analysed with the Freundlich, ${ }^{46}$ Langmuir-Freundlich or also called Sips ${ }^{47}$ Toth, ${ }^{39,40}$ and Redlich-Peterson ${ }^{48}$ expressions. The continuous distribution functions assume the adsorbent contains an infinite number of different types of binding sites, where $\mathrm{Eq}(3)$ and $\mathrm{Eq}(4) \mathrm{can}$ be replaced by: ${ }^{28}$

$$
q=\int_{-\infty}^{\infty} \frac{N(K) K C_{e}}{1+K C_{e}} d(\log K)=\int_{0}^{\infty} \frac{(0.4343) N(K) C_{e}}{1+K C_{e}} d K
$$

$N_{0}=\int_{-\infty}^{\infty} N(K) d(\log K)$

According to eq (7) and (8), the term $\frac{N(K) d(\log K)}{N_{0}}$ represents the probability of finding a site that will have an affinity between $\log \boldsymbol{K}$ and $\log \boldsymbol{K}+\boldsymbol{d}(\log \boldsymbol{K})$. Theoretically, $N(K)$ is called an affinity spectrum or affinity distribution function, which can be a function of $\log K$ or $K$. Theoretically, $N(K)$ refers to the affinity spectrum and is plotted against log $K$ to make it proportional to the binding energy, $\Delta G$. According to Hunston, ${ }^{28} N(K)$ can produce a continuous distribution spectrum that will closely simulate the affinity parameters that can be obtained from the discrete distribution models.

Expressions similar to eq (7) does frequently appear in literature in several scientific areas and within the context of adsorption, this expression represent the basic integral equation commonly used to describe the adsorption on heterogeneous surface. Eq (7), the Fredholm integral equation of first kind, is difficult to solve as it has no general analytical solution. Thus several attempts have been made to find an approximate solution and some of these methods were recently reviewed. Hunston used the finite difference method based on a method developed by Ninomiya and Ferry ${ }^{38}$ to estimate N(K). This technique can be easily applied by identifying a suitable continuous distribution model (like., Freundlich, Toth, Sips and RedlichPeterson isotherm) that best represent the experimental equilibrium data.

Eq (7), the Fredholm integral equation of the first kind, is difficult to solve as it has no general analytical solution. Thus, Hunston used a finite difference method based on a method developed by Ninomiya and Ferry ${ }^{38}$ to estimate $N(K)$. According to Hunston, the affinity distribution function, $N(K)$ is given by ${ }^{28}$

$$
N(K)=\left|\frac{q\left(a C_{e}\right)-q\left(C_{e} / a\right)}{2 \log a}-\frac{a}{(a-1)^{2}} \frac{\left[q\left(a^{2} C_{e}\right)-q\left(C_{e} / a^{2}\right)\right]-\left[q\left(a C_{e}\right)-q\left(C_{e} / a\right)\right]}{2 \log a}\right|_{K=\frac{1}{p}(o r) 1 / C_{e}}
$$

In Eq (9), $a>1$ is an arbitrary constant. This is the second-order approximation equation obtained by Ninomiya and Ferry. ${ }^{38}$ The value of $K=1 / p$, for gas phase adsorption and for the case of liquid phase adsorption, $K=1 / C_{\mathrm{e}}$. To calculate the affinity distribution function, it is essential to find a suitable isotherm model that can accurately reproduce the experimental equilibrium data. For example, the Freundlich isotherm assumes the binding affinities of the different types of adsorption sites are exponentially distributed. The Langmuir-Freundlich isotherm and the Toth isotherm assume the binding affinities of the adsorption sites will follow a quasi-Gaussian distribution.

In this work, as a case study, we used only the three-parameter Toth isotherms to describe adsorption on both homogeneous and heterogeneous surfaces. Mathematically, the Toth isotherm can predict the monolayer adsorption capacity of a homogeneous surface that will match with the surface area predicted by Langmuir isotherm. The Toth isotherm is given by $^{39,40}$

$q=\frac{q_{m} p}{\left(\left(1 / K_{t}\right)+p^{t}\right)^{1 / t}}$

where $K_{t}$ and $t$ are Toth isotherm constants. The value of $K_{t}$ is related to the binding energy. $q_{\mathrm{m}}$ is the monolayer adsorption capacity. The most frequently appearing expression is given by ${ }^{39,40}$

$q=\frac{q_{m} p}{\left(b+p^{t}\right)^{1 / t}}$

Page $\mid 8$ 
Where, $b=1 / K_{t}$. The value of $t$ measures the heterogeneity of the adsorbent surface and typically range from 0 to 1 . When, $t=1$, eq (11) reduces to a Langmuir isotherm.

Toth also proposed another expression that involves three isotherm constants given by: ${ }^{39,40}$

$q=\frac{q_{m} \chi^{1 / t} p}{\left(1 / K_{t}+p^{t}\right)^{1 / t}}=\frac{q_{m} \chi^{1 / t} p}{\left(b+p^{t}\right)^{1 / t}}$

where, $\chi$ is a constant and is given by

$\chi=\frac{1}{K_{t}}+1$

For the case of liquid phase adsorption, $p$ in $\mathrm{Eq}(11)$ to (12) will be replaced by the equilibrium concentration, $C_{\mathrm{e}}$.

The Toth isotherm can predict the relationship between $q$ versus $p$. Rewriting Eq (9) according to the Toth isotherms, as in $\mathrm{Eq}(11)$ and (12) to give the expressions in Eq (14) and (15), respectively.

$N(K)=2.5 \mid q_{h}\left(\frac{q_{m} a_{1}}{\left(b+\left(a_{1}\right)^{t}\right)^{1 / t}}\right)-q_{h}\left(\frac{q_{m}\left(a_{2}\right)}{\left(b+\left(a_{2}\right)^{t}\right)^{1 / t}}\right)-4.6\left[\frac{q_{m}\left(a_{3}\right)}{\left(b+\left(a_{3}\right)^{t}\right)^{1 / t}}-\frac{q_{m}\left(a_{4}\right)}{\left(b+\left(a_{4}\right)^{t}\right)^{1 / t}}\right]-\left[\frac{q_{m}\left(a_{1}\right)}{\left(b+\left(a_{1}\right)^{t}\right)^{1 / t}}-\right.$

$\left.\frac{\boldsymbol{q}_{\boldsymbol{m}}\left(\boldsymbol{a}_{2}\right)}{\left(\boldsymbol{b}+\left(\boldsymbol{a}_{2}\right)^{t}\right)^{1 / t}}\right]\left.\right|_{K=1 / C_{e}(\text { or }) 1 / p}$

$N(K)=2.5\left|\left(\frac{q_{m} \chi^{1 / t} a_{1}}{\left(b+\left(a_{1}\right)^{t}\right)^{1 / t}}\right)-\left(\frac{q_{m} \chi^{\frac{1}{t}} a_{2}}{\left(b+a_{2} t\right)^{\frac{1}{t}}}\right)-4.6\left[q\left(\frac{q_{m} \chi^{1 / t} a_{3}}{\left(b+\left(a_{3}\right)^{t}\right)^{1 / t}}\right)-q\left(\frac{q_{m} \chi^{1 / t} a_{4}}{\left(b+a_{4}\right)^{1 / t}}\right)\right]-\left[\frac{q_{m} \chi^{1 / t} a_{1}}{\left(b+a_{1}\right)^{1 / t}}-\frac{q_{m} \chi^{1 / t} a_{2}}{\left(b+a_{2}{ }^{t}\right)^{1 / t}}\right]\right|_{K=1 / C_{e} \text { (or) } 1 / p}$

(15)

For liquid phase adsorption, $a_{1}=a C_{\mathrm{e}}, a_{2}=C_{\mathrm{e}} / a, a_{3}=a^{2} C_{\mathrm{e}}$ and $a_{4}=C_{\mathrm{e}} / a^{2}$; and for gas phase adsorption, $a_{1}=a p, a_{2}=p / a, a_{3}$ $=a^{2} p$ and $a_{4}=p / a^{2}$.

Once the Toth isotherm parameters are determined using Eq (11) and (12), then the affinity spectra, $N(K)$ can be calculated from $\mathrm{Eq}(14)$ and $\mathrm{Eq}$ (15), respectively. Both of these expressions will be valid for an assumed value of $a=10^{0.1}{ }^{28,37} \mathrm{Eq}(14)$ and (15) can predict the number of binding sites $N$ and their affinity $K$ towards a specific solute molecule based on the Toth isotherm that relates $q$ with $C_{e}$. In both of these expressions, the binding affinity, $K$ is related to the equilibrium concentration, $C_{\mathrm{e}}$ by the relation, $C_{\mathrm{e}}=1 / K$.

\subsection{Non-Linear Regression Analysis}

The accuracy of the affinity spectrum, $N(K)$ depends on the accuracy of the determined isotherm parameters. Because the Toth isotherm contains more than two parameters, the ideal approach is to use a non-linear regression analysis, where the isotherm parameters can be determined using a suitable iterative technique by minimizing the error distribution between experimental data and the predicted isotherm. For non-linear regression, a trial and error method was developed using the solver add-in available within Microsoft Excel. The error distribution was minimized via a suitable error function; in this study, sum of the errors squared (ERRSQ) (see Table 3 of the supplementary file) was used as the error function and set as the objective function while determining the isotherm parameters. The objective function was set to reach its minimum while performing the iterations using the solver add-in function. Higher the ERRSQ, higher is the difference in the error between the experimental equilibirium data and the predicted isotherms. The value of ERRSQ near to zero means the difference in the error between the experimental data and the predicted isotherms is negibilge. To determine the Toth isotherm parameters the experimental data were fitted in the linearized version of the Langmuir isotherm (eq (2)) and the Langmuir isotherm parameters were determined using a simple linear-regression analysis. The calculated Langmuir isotherm parameters, $N$ and $K$ are assigned as initial guess values for the Toth parameters, $q_{\mathrm{m}}$ and $K$. Additionally, $t=0.5$ was set as the initial guess Page | 9 
value while implementing the non-linear regression algorithm. Additonal details about the non-linear regression method used in this work can be found elsewhere. ${ }^{49}$

A non-linear regression analysis was used to determine the binding affinity parameters in the discrete models like biLangmuir and tri-Langmuir isotherms. In this case, the initial values for the affinity parameters (number of adsorption sites and their binding affinity) were determined from the slope and intercept of the Scatchard plot. The determined values were then used as initial guess values for the non-linear regression analysis. Again, the sum of the squared errors was used as the error function to minimize the error distribution. Non-linear regression analysis always produced binding affinity parameters that are comparable to that of the ones determined using the affinity spectrum obtained using the continuous distribution (Toth) model. Thus, in this work, irrespective of the model (discrete or continuous) the binding affinity parameters were calculated using only non-linear regression analysis. In the supplementary file, we showed how to obtain the isotherm paramters in a multi-site Langmuir isotherm and Toth isotherm using the non-linear regression analysis.

\subsection{Binding Affinity Distribution according to a Toth Isotherm}

Before applying the Toth isotherm, the significance its parameters in $\mathrm{Eq}(11)$ and $\mathrm{Eq}$ (12) on the binding affinity distribution using Eq (14) and Eq (15), respectively, was tested. To do this, the affinity spectrum for different assumed values to Toth parameters was generated. Figure 4a shows the plot of $K$ versus $N(K)$ obtained using Eq (11) for a fixed maximum number of adsorption sites, $q_{\mathrm{m}}=30.8$ and $K_{\mathrm{t}}=1 / b=1$. When the heterogeneity parameter $t=1$, the Toth isotherm exhibits a quasiGaussian distribution with a broadening at high adsorption energies, a typical shape of an affinity distribution spectra for homogeneous adsorbents. The $x$-axis is plotted in the units of $\log (K)$ instead of $N(K)$. The term $\log (K)$ is proportional to the Gibbs free energy, $\Delta \mathrm{G}$ and thus often the affinity spectra is called as site-energy distribution or site energy spectra. Figure $4 \mathrm{~b}$ plots the affinity distribution in terms of $N(K)$ versus $K$ for a homogeneous material (blue line) and a heterogeneous material (red-line). For convenience, $K$ is plotted in logarithmic scale. The heterogeneity was introduced into the affinity spectra (red-line) by assuming $t=0.6$. The affinity spectra can be divided into two regions, the unimodal peak region (thick lines) and the exponentially tailing distribution (thin lines). These two regions can be related to the experimentally measurable adsorption isotherms shown in Figure $4 \mathrm{c}$. Figure $4 \mathrm{~b}, 4 \mathrm{c}$ show how the different regions in the affinity spectrum can be related to the experimentally measurable equilibrium data. At very low equilibrium concentrations (thinner lines in Figure $4 \mathrm{~b}$ and 4c), the adsorption sites with high binding affinity alone will be sampled and thus the affinity distribution spectrum will follow an exponential decaying distribution. Thus, the exponentially tailing distribution can be attributed to the sub-saturation region in the adsorption isotherm.. ${ }^{18,27,50,51}$

In these cases, it is very difficult to reach saturation with a low solute concentration in the bulk solution due to the surface heterogeneity and only the adsorption sites with high affinity can be sampled. The unimodal peak, with a peak maximum, corresponds to the sites sampled at higher loadings (the saturation region; thicker lines in Figure $4 \mathrm{~b}$ and $4 \mathrm{c}$ ). This type of affinity spectra is common if the adsorbent contains sites with narrow and homogeneous distribution of binding energy or binding affinities. If the material is homogeneous and contains binding sites with very high affinity towards the target molecule, then all the sites can be sampled within a smaller concentration or pressure range. The unimodal affinity distribution with a peak followed by the exponential tail can also signify a heterogeneous adsorbent if the distribution is broader. The broader the distribution implies that there are a greater number of different types of adsorption sites, with a wide range of binding affinities. If the material is highly heterogeneous, then in some cases it is more likely that the unimodal peak region cannot be sampled and the distribution spectra will be just exponential. The combination of unimodal distribution followed by exponentially tailing distribution can be taken as universal and can predict the affinity spectra of several adsorbents. 

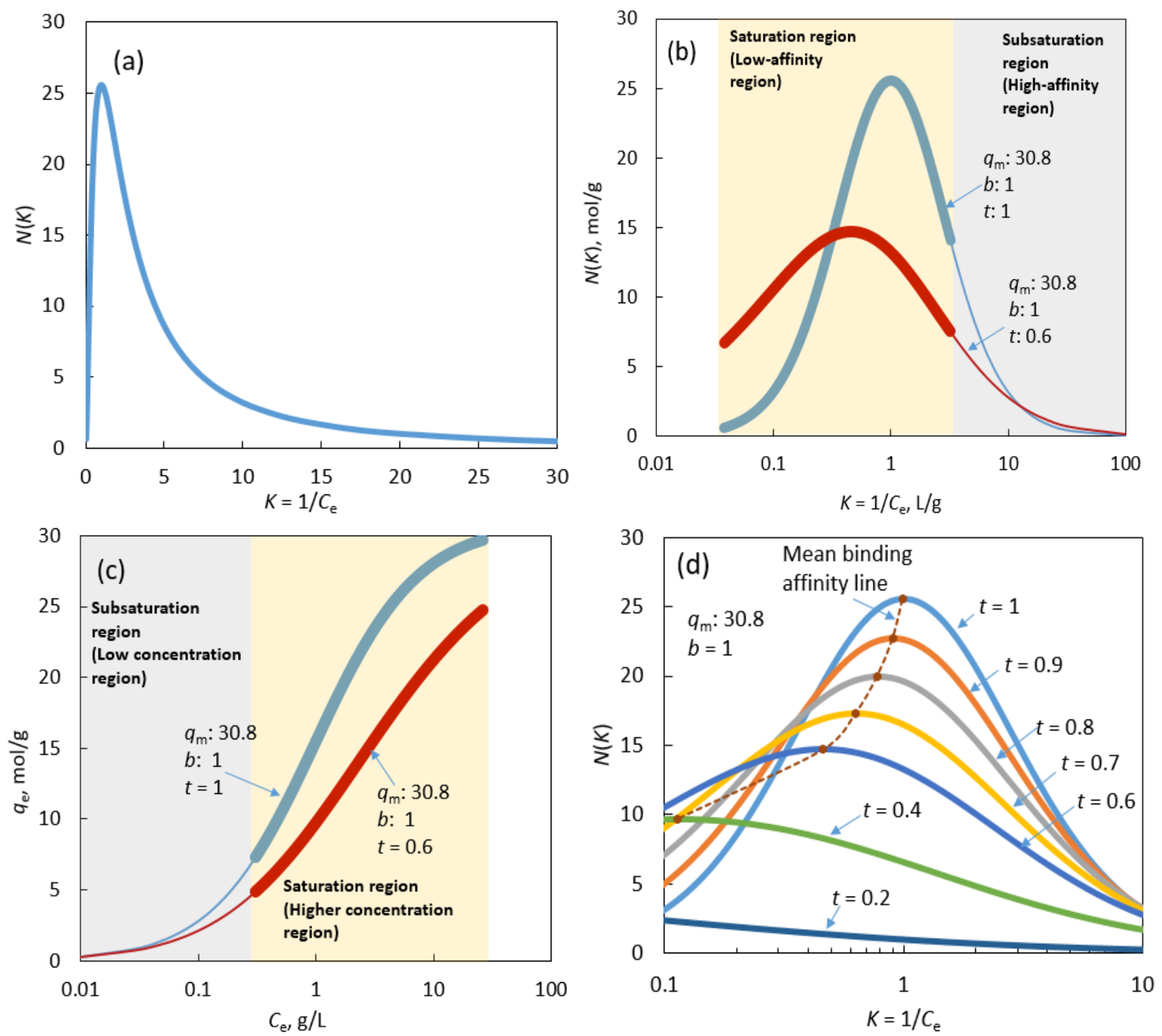

Page | 11 

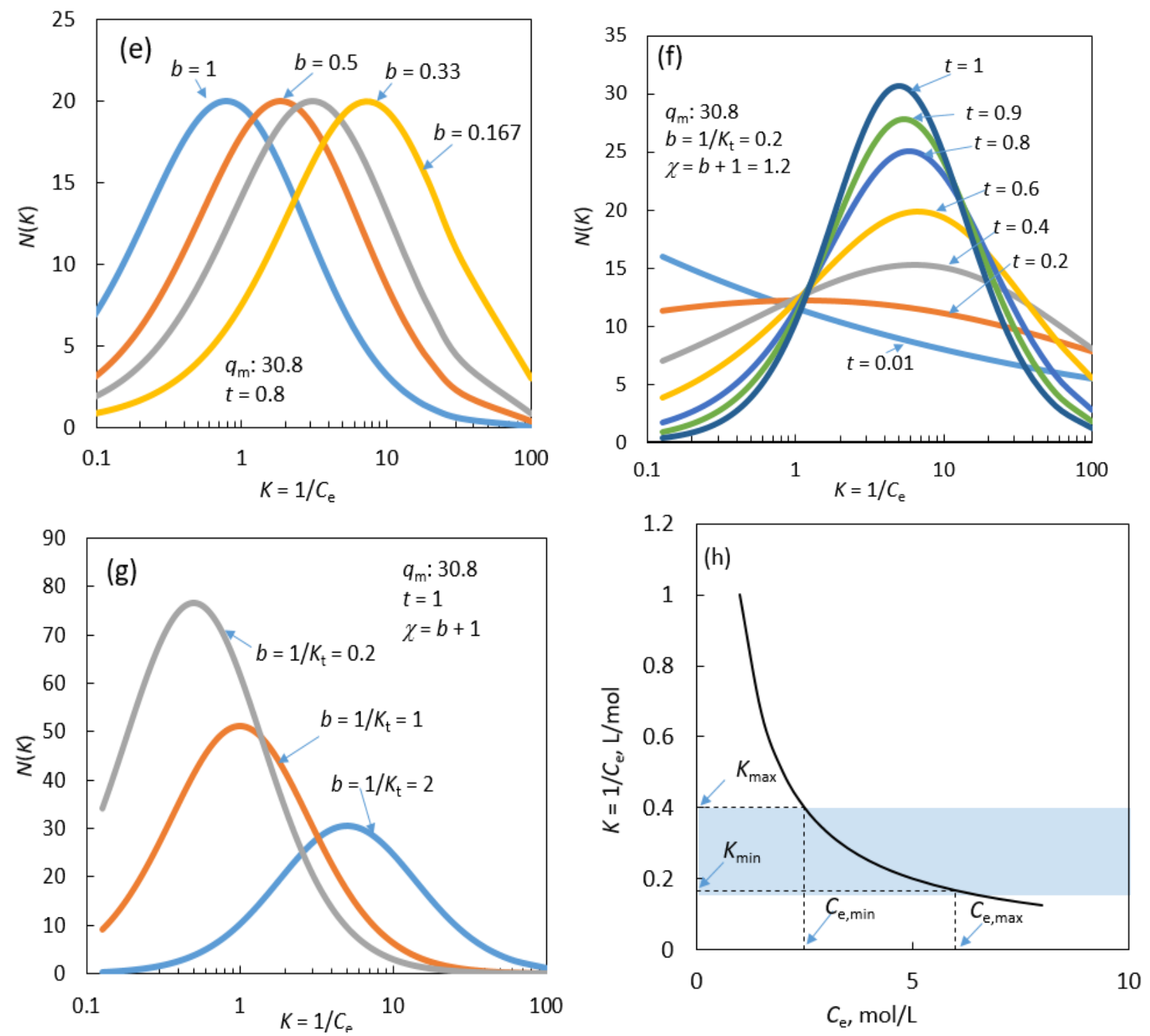

Fig. 4 (a) Quasi-distribution of binding affinities in a homogeneous adsorbent according to the Toth isotherm (Eq (11)). Site affinity spectra obtained using $\mathrm{Eq}(14)$ with $q_{\mathrm{m}}=30.8, b=1$ and $t=1$. (b) Affinity spectra of a homogeneous and heterogeneous adsorbent according to the Toth isotherm as in Eq (11); affinity spectra were obtained using Eq (14). Thick lines correspond to the affinity distribution of sites with low binding affinity and thin lines correspond to the affinity distribution of sites with a higher binding affinity. (c) Toth adsorption isotherm of a homogeneous and heterogeneous adsorbent. (d) Influence of the Toth heterogeneity parameter, $t$, in Eq 11 on the shape of the affinity spectra for a fixed adsorption capacity, $q_{\mathrm{m}}=30.8$ and $b=1$. The dotted lines show the change in the main binding affinity as a function of the heterogeneity parameter, $t$. (e) influence of the Toth isotherm parameter, $b$, in Eq 11 on the shape of the affinity spectra for a fixed adsorption capacity, $q_{\mathrm{m}}=30.8$ and $t=0.8$; (f) influence of the Toth heterogeneity parameter, $t$, in Eq 12 on the shape of the affinity spectra for a fixed adsorption capacity, $q_{\mathrm{m}}=30.8, b=1 / K_{t}=1$ and $\chi=b+1=2 ;(\mathrm{g})$ influence of the Toth heterogeneity parameter, $b$, in Eq 12 on the shape of the affinity spectra for a fixed adsorption capacity, $q_{\mathrm{m}}=30.8, b=1 / K_{t}$ $=1$ and $\chi=b+1=2$ and $(\mathrm{h})$ relationship between the binding affinity and the equilibrium concentration in the experimental adsorption isotherm.

This concept was tested by generating the affinity spectra using Eq (14) for fixed $q_{\mathrm{m}}$ and $b$ values but for different values of $t$ (Figure $4 \mathrm{~d}$ ). The shape of the affinity distribution broadens with the heterogeneity parameter, $t$, decreasing from 1 to 0.2 . Page | 12 
When $t=0.05$, the affinity distribution follows an exponentially decaying trend. The decaying exponential dependence also indicates the adsorbent contains a relatively higher concentration of sites with low binding affinity than adsorption sites with higher binding affinity; this is a typical surface property of a heterogeneous adsorbent. Another notable feature is that the mean binding energy (the peak maximum) decreases with decrease in the heterogeneity parameter, $t$. The mean binding energy decreases from $K=0.99$ to 0.113 for a decrease in the heterogeneity parameter from $t=1$ to $t=0.4$. The Toth isotherm generated with constant $q_{\mathrm{m}}$ and $t$ but for varying $b$ values (see Figure 4e) shows the width of the distribution is not influenced by $K$ values; however, the mean binding affinity tends to increase with an increase in $K$ values.

Likewise, the affinity spectra of the Toth isotherm was calculated as in Eq (15) using the numerical method. The affinity spectra were generated for fixed values of $q_{\mathrm{m}}=30.8, b=0.2$ but for different $t$ values ranging from 0.1 to 1 (Figure $4 \mathrm{f}$ ). Increasing the $t$ value increases the width of the affinity distribution. The $t$ value near to unity shows a much narrower distribution of binding site affinities, a characteristic property of homogeneous adsorbents. Increasing $t$ results in a minor shift in the mean binding energy. The mean binding energy decreases with an increase in $t$ value from 0.2 to 1 . When $t=$ 0.01, the Toth expression as in Eq (15) (and for the assumed $q_{\mathrm{m}}$ and $b$ values) produced exponentially decreasing binding affinities, a characteristic property of heterogeneous materials. Figure $4 \mathrm{~g}$ shows the affinity distribution calculated from Eq (15) for different values $b$ and fixed values of $q_{\mathrm{m}}$ and $t$. Clearly, increasing the $b$ values decreases the mean binding energy significantly with a noticeable difference in the broadening of the affinity distribution. The lower the $b$ value, the lower the concentration of adsorption sites with affinities equivalent to the mean binding energy and vice versa.

Figure $4 \mathrm{~d}-4 \mathrm{~g}$ shows how the affinity spectra will vary depending on the Toth parameters. This type of information is crucial for adsorbent design. Both Eq (14) and (15), can successfully capture the heterogeneity of most of the adsorbents. Figure 4df clearly shows for a combination of the Toth parameters, generating affinity spectra is straightforward from the Toth isotherm parameters.

It is should be mentioned that both Eq (14) and (15) have no mathematical or theoretical limitations. The affinity spectra can be generated with binding affinity parameters ranging from zero to infinity. Thus, the affinity spectra, $N(K)$ estimated only within the analytical limits $K_{\min }$ and $K_{\max }$, should be considered to be valid for analytical purposes. These analytical limits can be set based on the minimum and maximum concentration or pressure in the experimental isotherm:

$K_{\min }=1 / C_{\mathrm{e}, \max }$ and $K_{\max }=1 / C_{\mathrm{e}, \min }$

For gas-phase adsorption, the $K_{\min }$ and $K_{\max }$ will be related to the minimum and maximum pressures in the experimentally measured adsorption isotherm.

$K_{\min }=1 / p_{\max }$ and $K_{\max }=1 / p_{\min }$

Figure $4 \mathrm{~h}$ plots the relationship between the equilibrium concentration and the binding affinity. Affinity spectra can nominally be calculated above or below $K_{\min }$ and $K_{\max }$; however, the number of adsorption sites associated with a binding affinity greater than $K_{\max }$ or lower than $K_{\min }$ may not be the actual characteristic property of the material. Another key issue that should be remembered is the units of $N(K)$ and $K$. If the equilibrium concentration is expressed in terms of mol $\mathrm{L}^{-1}$, then the binding affinity is expressed in terms of $\mathrm{L} \mathrm{mol}^{-1} . N(K)$ and the amount adsorbed at equilibrium has the same units. If the amount adsorbed is expressed in terms of $\mathrm{mol} \mathrm{g}^{-1}$, then $N(K)$ generated according to Eq (14) and (15) will have the same units (i.e., $\mathrm{mol} \mathrm{g}^{-1}$ ).

\section{Applicability of Toth Isotherms for The Characterisation of Adsorbents}

\subsection{Characterisation of molecularly imprinted polymers (MIPs)}

Molecularly imprinted polymers (MIPs) are custom made materials that can recognise molecules and thus widely used as gas and liquid sensors, adsorbents, chromatographic stationary phases, affinity assays, catalysis and chiral separations. ${ }^{22-}$

Page | 13 
24,50,52-54 They are also porous with surface areas ranging from $\sim 50-150 \mathrm{~m}^{2} \mathrm{~g}^{-1}$. MIPs are synthesised by creating a complex template with a functional monomer to form either a covalent or a noncovalent pre-polymerisation complex (Umpleby et al., Anal Chem). ${ }^{29,45,55}$ A cross-linking agent, added in excess, is then polymerized with this adduct. Finally, the template is removed from the densely cross-linked matrix, which leaves the rigid bulk material with a cavity of size and shape that should be commensurate with the shape of the template molecule. ${ }^{24,50}$ In the ideal case, the imprinting process should leave the material with only one type of binding sites or cavity that fits the target molecule. However, most laboratory-synthesised materials do not exhibit this idealised property and often contain binding sites that are indifferent to that of the target molecule. The final material contains a relatively broad distribution of binding sites with a wide range of binding affinities. ${ }^{53}$ Heterogeneity is considered to be an undesirable property of MIPs and is considered to be a prime contributor of broad and asymmetric elution peaks in chromatographic applications. ${ }^{56}$ Heterogeneity also limits the sensitivity of sensors and decreases the linearity in immunoassay-type analyses. ${ }^{57,58}$ Thus, the applicability of the Toth isotherm to characterise the heterogeneity of MIPs, at least 13 different isotherms are selected. These isotherms include MIPs obtained via non-covalent (1-2, 4-13) and covalent imprinting techniques (3), different polymerisation conditions, different monomers, and photochemically (1-3) and thermally (4-13) initiated polymerisation conditions. These MIPs are selective for ethyl adenine9-acetate (1), 9-ethyladenine (2), ${ }^{45},{ }^{59}$ cholesterol (3), ${ }^{55}$ diclofenac (4-7), ${ }^{54}$ mefanamic acid (8-11), ${ }^{54}$ and sinapic acid (12-13). ${ }^{60}$ of the associated isotherms were selected to span a range of concentration limits. A few of the selected adsorption isotherms cover only the adsorption at lower equilibrium concentration, which is far away from the equilibrium concentration where complete saturation of the adsorption sites can be expected. This means only the adsorption sites with the highest affinities are covered by the target molecules and thus they cannot capture the distribution of affinities of the adsorption site with low binding affinities.

A few of the adsorption isotherms measure the adsorption over a wide range of equilibrium concentrations up to the saturation point. The experimentally obtained equilibrium data are taken from the literature and fitted to the Toth isotherms to obtain the Toth isotherm parameters. The Toth isotherm successfully predicts the experimental equilibrium data, thus allowing their affinity distribution to be predicted (see Table 1 of supplementary file).

Figure 5a, shows the affinity distribution of the three most studied and well characterised MIPs, 1-3. The affinity distribution was obtained from Eq (14) and the Toth parameters were obtained by fitting the experimental data taken from the literature with a Toth isotherm using a non-linear regression analysis technique described above. The Toth isotherm parameters of MIPs 1-3, and the other systems studied in this work, are given in Table 1 of the Supplementary file. MIP 2 that can bind adenine was originally synthesised by Shea et al., ${ }^{59}$ MIP 3 that can bind cholsterol was synthesised by Whitcombe et al. ${ }^{55}$ using the covalently imprinting technique. Whitecombe et al. implemented a unique sacrificial spacer imprinting process resulting in material with only one type of adsorption site, i.e., this material should be homogeneous. MIP 1 was originally synthesised by Umpleby et al., ${ }^{45}$ with a composition identical to MIP 2 and adsorption spaces are obtained using the noncovalently imprinting technique. The only difference between MIP 1 and 2 is the imprinting solvent, chloroform for 2 and acetonitrile for 1. Earlier, Umpleby et al. used a Langmuir-Freundlich expression and a numerical technique to characterise these materials and showed MIP 1 and 2 are heterogeneous and MIP 3 is homogeneous. ${ }^{45}$ Whitecombe et al., used a Scatchard plot to show MIP 3 contains only one type of adsorption site. ${ }^{55}$ These three polymers were deliberately chosen becuase their binding parameters were already characterised using different approaches and thus they can serve as benchmark materials to test the efficacy of the Toth models to calculate the affinity spectra.

The results presented in Figure 5a agree with earlier observations. ${ }^{45,55,59}$ According to the Toth isotherm, the non-covalently imprinted polymers, 1 and 2 contain a more heterogeneous distribution of binding affinities and they show an exponentially decaying distribution with a higher concentration of adsorption sites with low binding affinity. The covalently imprinted polymer 3 exhibited the expected homogeneous distribution of binding affinities with a maximum at $1.69 \mathrm{mM}^{-1}$. The affinity distribution of 1 and 2 are similar and this is due to the nearly identical properties of these MIPs in terms of composition, template and stoichiometry. ${ }^{55,55}$ These results are in agreement with the previous work of Umpleby et al. ${ }^{55}$ Their characterisation studies confirmed that MIPs 1 and 2 are heterogeneous with identical binding affinity distribution and that MIP 3 is homogeneous. The only difference we observed in this work is that for the case of MIP 3, the concentration of the adsorption sites with binding affinities equivalent to the mean binding energy is relatively higher than the one reported by

Page | 14 
Umpleby et al. ${ }^{45}$ This slight difference is likely due to the difference between the methods used to generate the affinity spectra. Umpleby et al., used an empirical relation obtained from the plot of $\log q_{\mathrm{e}}$ versus $\log C_{\mathrm{e}}$ and in this work the Toth isotherm is used to predict the relationship between $C_{\mathrm{e}}$ versus $q_{\mathrm{e}}$.

For comparison, the affinity spectra of the MIPs, 2 and 3 were calculated using the Eq (15), which is based on the Toth isotherm in Eq (12) and is shown in Figure 5a (dotted lines). Eq (15) produced affinity spectra that almost overlap with the ones obtained using eq (14). In particular, for polymer 2, the affinity spectra obtained from Eq (14) and (15) are overlapping, showing negligible difference between the calculated affinity spectra based on the two different Toth expressions. Both of these Toth expressions captured the essential properties of MIPs 2 and 3; 2 with heterogeneous distribution of binding affinities and 3 containing a homogeneous distribution of binding affinities. Clearly, both of the Toth expressions can be used to characterise the MIPs for their binding affinities towards a specific target molecule. As both of these expressions essentially produce the same results and produce similar information about the binding properties of the MIPs, in the rest of the work only Eq (14) was used for convenience to characterise more MIPs and other classes of adsorbents.
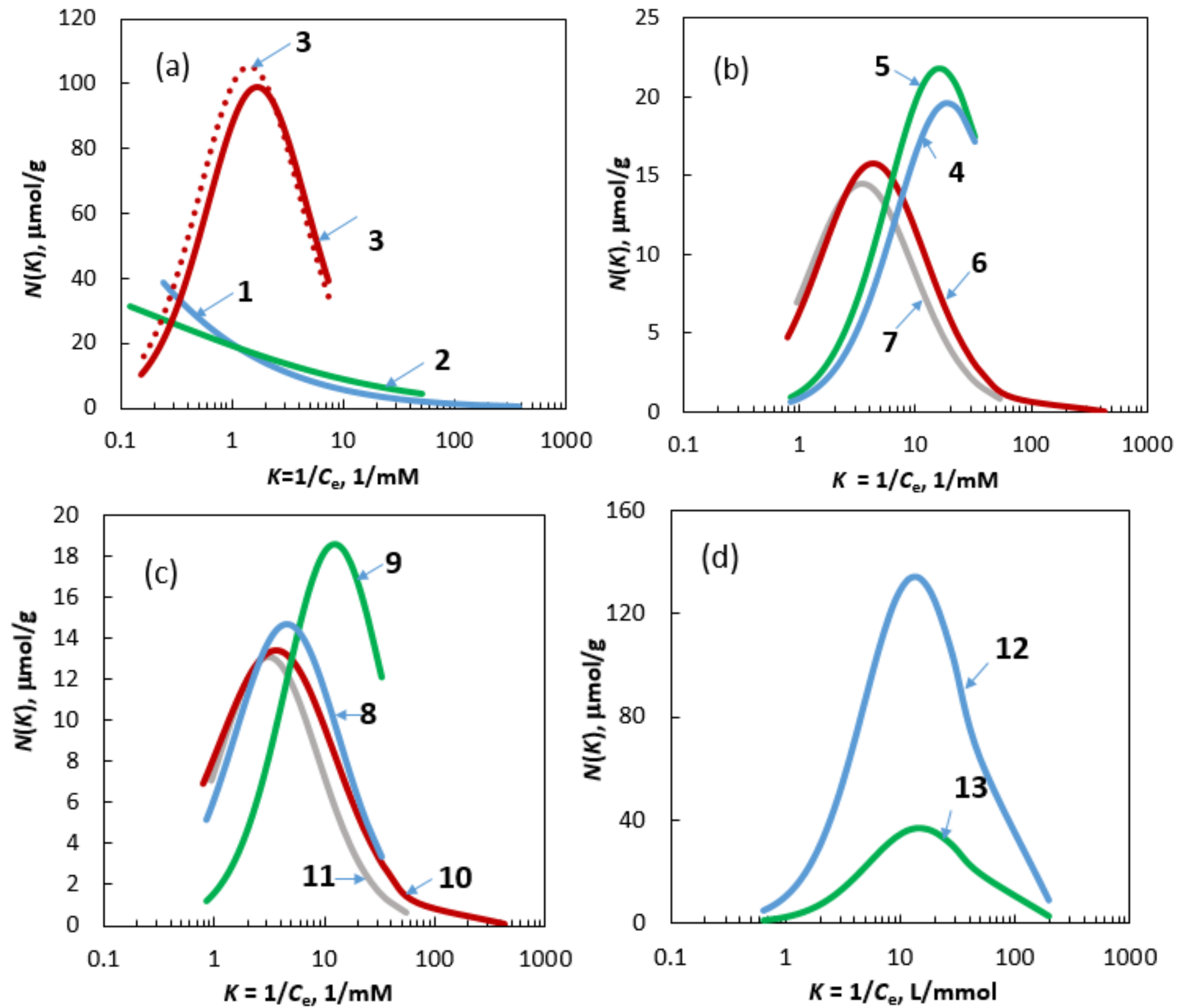

Page | 15 
Fig. 5 (a) Affinity spectra of MIPs 1-3; (b) affinity spectra of diclofenac recognizing MIPs 4 -6 (c) affinity spectra of mefanamic acid recognizing MIPs 8-11 and (d) affinity spectra of sinapic acid recognizing MIPs 12 and 13; the bars show the affinity spectra obtained using the Scatchard plot method. The solid lines correspond to the affinity spectra obtained using Eq 14 and dotted line corresponds to the affinity spectra obtained using Eq 15.

To show the general applicability of the Toth isotherm, eight more MIPs (polymers 4-11) synthesised by Anfossi et al. ${ }^{54}$ were characterised (Figure $5 \mathrm{~b}$ and $5 \mathrm{c}$ ). The molecular level pore spaces in these polymers are created using a non-covalent imprinting technique. The authors implemented a new strategy to alter the binding properties by delayed addition of the template molecules. ${ }^{54}$ Polymers 4, 5, 6 and 7 were synthesised by adding the template diclofenac at $t=0,10,20$ and 30 min, respectively from the start of polymerisation. ${ }^{54}$ Polymers $8,9,10$ and 11 were synthesised by adding the template mefanamic acid $t=0,1020$ and 30 min, respectively from the start of polymerisation. ${ }^{54}$ This system was specifically chosen in order to expose the changes in binding properties of the MIPs as a function of time at which the template molecules were added. In general, the polymers can recognize the guest molecules as evidenced by their unimodal distribution of the binding affinity. For the case of diclofenac, all the polymers, except 6, exhibit a homogeneous distribution of binding sites; polymer 6 shows a slightly broader peak. The imprinted polymers 4-7 specifically bind mefanamic acid. Polymers 8-11 specifically binds diclofenac. Figure $3 \mathrm{~b}$ shows polymers 4 and 5 are almost identical with similar binding affinity. Polymer 7 and 8 contain identical binding properties towards diclofenac, although polymer 7 shows a slightly broader spectrum, signifying they are slightly heterogeneous. The mean binding affinity of 4 and 5 were higher than then mean binding affinity of the adsorption sites in 6 and 7. Additionally, the mean binding affinity of polymer 5 is slightly higher than 4 and the concentration of all the adsorption sites with different binding affinity is slightly higher than the ones in polymer 4 . This signifies that the addition of the templates after $10 \mathrm{~min}$ leaves a noticeable imprinting effect. However, the addition of templates after 20 and 30 minutes significantly diminished the imprinting effect and also the mean binding affinity.

For the case of mefanamic acid (Figure 5c), adding the sacrificial template after 10 minutes produced a noticeable imprinting effect in polymer 9; the binding affinity distribution is relatively higher than the affinity distributions of 8,10 and 11 . In fact, adding the template after 20 and 30 minutes worsened both the imprinting effect and the mean binding affinity, as observed from their relatively lower binding affinity distribution of the binding sites. Out of polymers 8, 9, 10 and 11, the polymers 8 and 10 are to be slightly more heterogeneous, as observed from their slightly broadened peak width when compared to other structures. Altering the content of cross-linker is considered to be an accepted strategy to tune the binding affinity and improve the molecular recognition capability of MIPs. It is essential to have information about the degree-linking on the imprinting effect. Thus, two different MIPs were selected, polymer 12 and polymer 13 synthesised originally by Til et al. ${ }^{60}$ using a sinapic acid template. The degree of cross-linking in polymer 12 was higher than in polymer 13 . In addition, the surface area of polymer $12\left(79.2 \mathrm{~m}^{2} / \mathrm{g}\right)$ is higher than the surface area of polymer $13\left(71.7 \mathrm{~m}^{2} / \mathrm{g}\right)$. Characterising these two polymers demonstrates the usefulness of the Toth isotherm to elucidate the effect of the degree of cross-linking on the imprinting efficacy. Figure 5d shows the site energy distribution spectra of polymer 12 and 13 . Both of these polymers appear to be homogeneous and exhibit a quasi-Gaussian/uniform distribution with a maximum at 12.5 and $15.38 \mathrm{~L} \mathrm{mmol}^{-1}$, respectively. This analysis shows means, irrespective of the degree of polymerisation, both polymers recognize sinapic acid and the only difference is the number of sites in 13 is significantly lower than 12 . It should be mentioned here that, polymers 4-7 was synthesised using diclofenac as template and also tested for the molecular recognition capability of a structrually similar compound mefanamic acid. The labels, polymer 8-11 was used here only for for the sake of convenience.

\subsection{Characterisation of benchmark MOFs and carbons used for methane adsorption using Toth isotherms}

To investigate the general applicability of Toth isotherms and the potential far characterisation other classes of porous material, the affinity distributions of two MOFs Al-soc-MOF1 Ref $\left({ }^{61}\right)$ and MOF-905 Ref $\left({ }^{62}\right)$ for methane were calculated. In terms of pore properties, both of these materials have a very large surface area of $5585 \mathrm{~m}^{2} \mathrm{~g}^{-1}$ and $3490 \mathrm{~m}^{2} \mathrm{~g}^{-1}$ with pore volume of 1.34 (Al-soc-MOF1) and $2.3 \mathrm{~cm}^{3} \mathrm{~g}^{-1}$ (MOF-905). These two materials are also considered due to their importance for natural gas storage: both of these materials can meet the DOE target and store and deliver at least 264 volume of methane 
in one litre of adsorbent material. ${ }^{4,63}$ An estimation of the binding affinity of these MOFs should, therefore, provide a general idea about the required level of binding affinity properties of storage materials that can meet the DOE targets and this can serve as a guideline in material design. In terms of physical properties, these MOFs have high specific surface area and pore volume when compared to the MIPs discussed in the previous section. In both of these materials, the total adsorption of methane was found to increase continuously with respect to the equilibrium pressure. This is expected, as adsorption experiments were performed at $298 \mathrm{~K}$; at this temperature methane is supercritical and thus there will be no condensation pressure. Thus, once the molecules filled the surface they continuously tend to occupy the void spaces (which might act like adsorption spots) available within the pore volume. The gas adsorbed within the pore-volume usually fills with a gas-like density and the binding affinity at this stage should be equal to fluid-fluid interactions. The determined Toth isotherm parameters theoretically allows the estimation of the affinity distributions irrespective of the this underlying adsorption mechanism. Figure 6 shows the affinity distribution of Al-soc-MOF1 and MOF-905. In the original works, adsorption isotherms were measured up to 80 bar; however, the binding affinity spectra were calculated as if the adsorption isotherms were recorded up to 250 bar since this is the pressure of conventional natural gas storage tanks.

The affinity distribution spectra clearly show both of these MOFs are homogeneous as evidenced by the smooth quasiGaussian distribution of the adsorption sites. It should be mentioned that the affinity spectra were generated over such a wide range of binding affinity to capture the theoretical fact that both of these adsorbents are homogeneous. However, if we consider the affinity distribution spectra within the analytical window set by the minimum and maximum pressure in the adsorption isotherm, $K_{\min }=1 / p_{\max } \sim 1 / 80=0.0125 \mathrm{bar}^{-1}$ and $K_{\max }=1 / p_{\min } \sim 1 / 0.01=100 \mathrm{bar}^{-1}$, both of the MOFs showed an exponential distribution of binding sites (see the $K_{\min }$ line in Figure 6 ). This does not mean these materials are heterogeneous, rather this should be taken as a result of the fact that more methane molecules are adsorbed onto adsorption sites with binding affinities that are less than the mean binding affinity of this material for methane. In fact, the determined heterogeneity parameter of the Toth isotherm, $t=1$ (see Table 1 of the Supplementary file) confirmed both of these adsorbents are homogeneous which can be expected from their highly periodic structure. The exponentially decaying distribution of affinities also means the adsorption sites with affinities less than the mean binding affinity are sampled by the adsorbing methane molecules at the studied pressure ranges. It should be noted that both MOF-905 and Al-Soc-MOF1 are structurally different with different surface chemistry and pore topology. The slight difference in the affinity distribution spectra attests to the different structure of the two materials. MOF-905 contains slightly higher concentration of binding sites with higher binding affinity. E.g., MOF-905 contains slightly more number of adsorption sites with binding affinity, $K>0.018$ bar $^{-1}$ (see Figure 6). Al-soc-MOF1 also contains a slighltly higher number of adsorption sites with lower binding affinity $(\mathrm{K}<0.018$ bar $\left.^{-1}\right)$. This means this MOF will adsorb more methane at higher pressures. Irrespective of these small differences observed in the affinity spectra, both of these MOFs deliver (deliverable capacity is usually defined as the adsorption at 80 bar minus adsorption at 5 bar) a similar amount of methane $\sim 194-200 \mathrm{v} / \mathrm{v}$. The combination of slightly higher number of adsorption sites with higher binding affinity $\left(K>0.018 \mathrm{bar}^{-1}\right)$ in MOF-905 seems to improve the methane deliverable capacity by a smaller amount. 


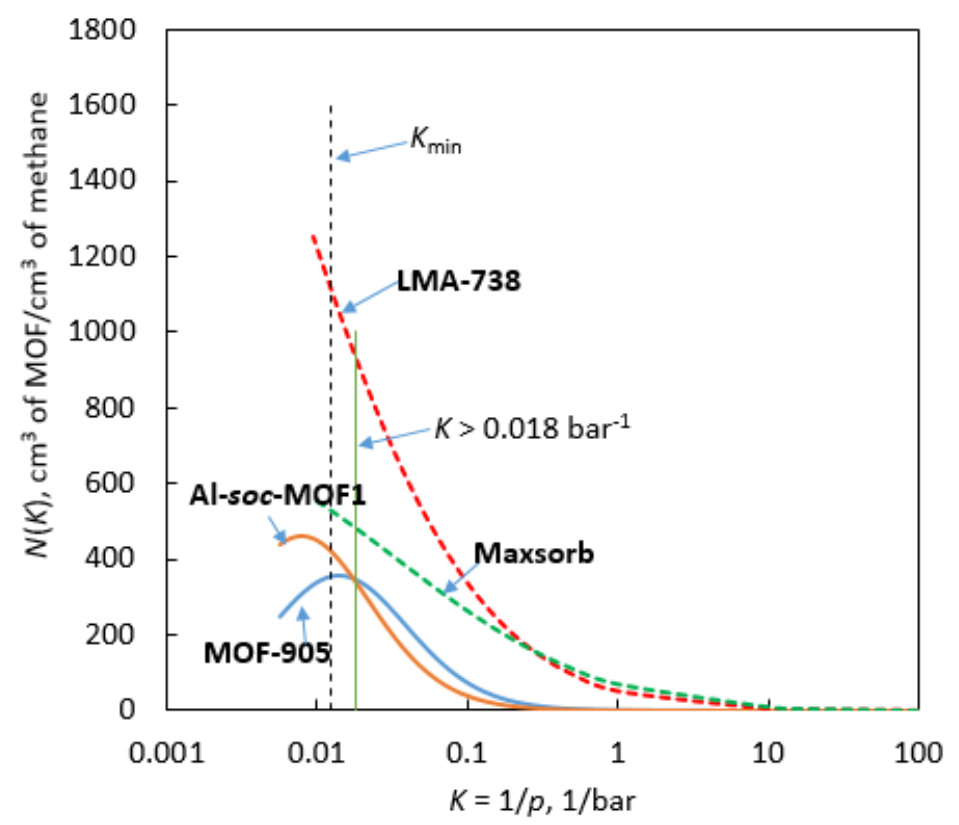

Figure 6: Affinity distribution spectra of two benchmark MOFs, best performing carbon material and a commercial activated carbon for the adsorption of methane at $298 \mathrm{~K}$.

The affinity distribution spectra of the two best MOFs were compared with a carbon-based material, LMA-738 (specific surface area $=3290 \mathrm{~m}^{2} \mathrm{~g}^{-1}$ and pore volume $=2.25 \mathrm{~cm}^{3} \mathrm{~g}^{-1}$ ), which is amongst the best performing material reported to date to store methane, and one of the most tested commercial carbon-based materials, Maxsorb (specific surface area $=1800 \mathrm{~m}^{2}$ $\mathrm{g}^{-1}$ and pore volume $=0.83 \mathrm{~cm}^{3} \mathrm{~g}^{-1}$ ). These materials differ structurally. Carbon-based materials contain a broader distribution of binding affinities when compared to MOFs, and are therefore far more heterogeneous. LMA-738 and Maxsorb contains higher number of binding sites than two of the MOFs.

In terms of volumetric capacity, at 80 bar and $298 \mathrm{~K}$, LMA-738 could store up to $965 \mathrm{v} / \mathrm{v}$ at $298 \mathrm{~K}$ (see Figure 1 in the Supplementary Information for the adsorption isotherms and the calculation procedures). This value is remarkably higher than the storage capacity of the best performing MOFs, Al-soc-MOF1 and MOF-905 which has a storage capacity of $~ 215$ $\mathrm{v} / \mathrm{v}$ and $200 \mathrm{v} / \mathrm{v}$, respectively. If Al-soc-MOF1 or MOF-905 is compared with LMA-738, it is clear that both of these MOFs contains a very low concentration of binding sites irrespective of their binding affinity. This explains the remarkably high storage value of methane observed in this carbon structure. Likewise, the Maxsorb, a commercial adsorbent, irrespective of the binding affinity, all the concentration of adstion sites on the surface of this material is significantly higher than the ones observed in MOFs. Clearly, the affinity spectra differentiate the material properties of the carbon based structures and two of the MOFs. It is clear that, unit volume of these carbon structures possess more number of adsorption sites irrespective of the type of adsorption sites when compared with two of the best performing MOFs. This particular material property makes LMA-738 and Maxsorb the ideal material for very high storage capacity for methane. The presence of the higher number of adsorption sites with higher binding affinity, together with a high concentration of adsorption sites with lower binding affinity, also favours the higher deliverable capacities of the MOFs. Figure 6 shows that, it is possible to express the affinity spectra in terms of the number of adsorption sites available per unit volume of adsorbent. This will be useful while screening porous material for their volumetric storage or deliverable capacities of gases.

\subsection{Characterisation of C18-bonded silica columns}

The Toth isotherm was used to characterise the affinity spectra of C18-bonded silica columns for phenol and caffeine. Phenol contains one polar group and one aromatic group, whereas caffeine contains two aromatic groups and many polar groups. Theoretically, the adsorption energy of caffeine onto a surface should be higher than the phenol due to its larger size and the additional number of sites that can interact with a surface. Additionally, the solubility of phenol is higher than caffeine. 
Typically, the retention of low molecular weight compounds on C18-bonded silica columns correlates with molecular weight and solubility. However, the experimental investigation performed by Gritti and Guiochon ${ }^{64}$ under similar experimental conditions with different silica columns showed caffeine elutes faster than phenol. To get a theoretical understanding on this experimental paradox, the binding affinity distribution spectra of three different columns were constructed: (i) one monomeric $\mathrm{C} 18$ bonded packed column (vydac), (ii) one polymeric C18-bonded packed column (phenomenex) and (iii) one monomeric bonded monolithic column (Merck). The caffeine and phenol adsorption isotherms in these columns were originally obtained by Gritti et al., using Frontal Analysis. ${ }^{64}$

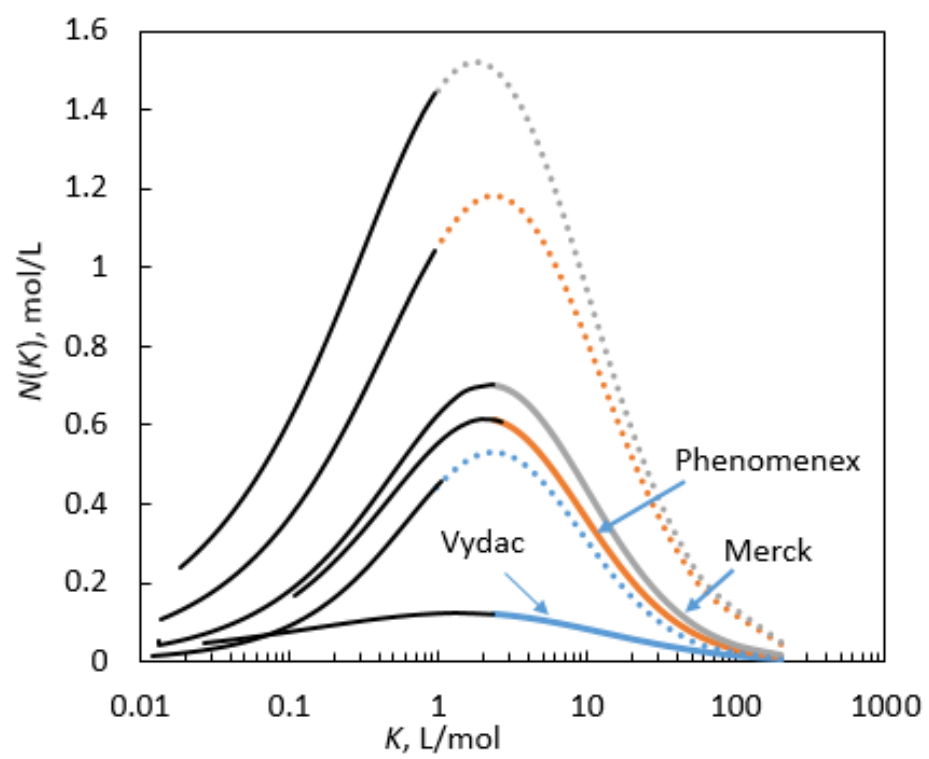

Fig. 7. Affinity spectra of three C18-bonded silica columns. The solid thick lines and the dotted lines correspond to the affinity distribution of the silica columns for caffeine and phenol (solid or dotted lines in blue: Vydac, orange: phenomenex and grey: Merck). Only the thick lines and the dotted lines can be taken as an accurate representation of the adsorbent properties as they correspond to the affinity distribution between the $K_{\min }$ and $K_{\max }$. The thin black lines correspond to extrapolated affinity distribution below $K_{\min }$.

The affinity spectra generated from the adsorption isotherms are shown in Figure 7. In the original works of Gritti et al., the adsorption isotherms were reported only in the sub-saturation regions, and thus the affinity spectra obtained using Eq (15) showed an exponentially decaying distribution. Thus in Figure 7, to compare the performance of the different columns within the same analytical window (binding affinity ranging from $K=0.02$ to $200 \mathrm{~mol} \mathrm{~L}^{-1}$ ), we also show the extrapolated affinity spectra obtained using the Toth isotherm parameters that best fit the experimentally measured equilibrium data (see the thinner black lines in Figure 7). Although the extrapolation of the affinity parameters outside the experimentally measured equilibrium concentration might lead to an erroneous distribution plot (see Figure 4h), this analysis demonstrates that most of the low affinity sites are left unoccupied by caffeine. The analysis also permits a theoretical indication that the caffeine molecules mostly occupy the adsorption sites with a binding affinity lower than the mean binding affinity of the adsorbent packed in the columns. It is clear from Figure 7 that all of these columns exhibit a quasi-Gaussian distribution of binding affinities, with a broadened peak, signifying that the packed adsorbents are slightly heterogeneous. Additionally, the solubility of phenol is higher than caffeine. Typically, the retention of low molecular weight compounds on C18-bonded silica columns correlates with molecular weight and solubility. The notable features in Figure 7 are: (i) at the experimental conditions studied, caffeine molecules are adsorbed onto sites with higher binding affinity and all the caffeine molecules are adsorbed onto the binding sites with an affinity greater than the maximum binding affinity (the peak maximum or the mean binding affinity) of the columns and (ii) the number of binding sites for caffeine is roughly two times lower than the number of binding sites of with similar binding affinity for phenol, (iii) irrespective of the binding affinity, the number of binding sites available for phenol is always higher than caffeine (in Vydac, binding site for caffeine is roughly four times lower than the binding sites available for phenol with similar binding affinity) and (iv) phenol moleclues are adsorbed on to both low 
and high affinity binding sites. The high number of sites available for phenol is expected because this molecule is approximately two times smaller than caffeine and this means more molecules can occupy per unit surface area of the materials in the packed column. The experimentally observed fact that caffeine elutes first can be attributed to the greater number of high binding affinity sites available for phenol. It is likely that the larger caffeine molecules are not accessible to all the available sites, with low binding energy due to pore diffusion resistance; whereas the smaller phenol molecules can easily diffuse through and access those high-affinity sites. This can be realised from Figure 7 since all the affinity spectra are generated within the same analytical window, allowing the material properties to be compared. A similar conclusion was proposed by Gritti and Guiochon based on the affinity parameters obtained using a simple Scatchard plot analysis and Henry's isotherm constant. ${ }^{64}$ Their Scatchard plot analysis confirmed that the saturation capacity of the low energy sites for phenol is about twice that measured for caffeine. Whereas the saturation capacity of the high-energy sites is about 15 times larger for phenol than for caffeine. They attributed the relatively lower retention time of caffeine with the relatively lower number of high-energy sites available for caffeine when compared to the relative intensity of adsorption constant measured using Henry's constant.

\subsection{Comparison of the continuous distribution spectra versus the discrete binding model}

The accuracy of the calculated affinity spectra using Toth isotherms was tested by comparing them with the spectra obtained using discrete models (Langmuir or multi-site Langmuir). To do this, the experimental equilibrium data of polymers 1, 2, 3, 12 and 13 in a tri-Langmuir plot was fitted using a non-linear regression technique assuming there exist at least three different types of binding sites on these adsorbents. We selected these MIPs only to demonstrate the difference between the affinity parameters obtained by a discrete model and Toth isotherm. For the case of polymers 1 and 2, non-linear regression analysis confirmed only a bi-Langmuir isotherm is enough to precisely model the experimental equilibrium data (the calculated isotherm parameters are given in Table 2 of the Supplementary file). For the case of polymers 3, 12 and 13, a simple Langmuir isotherm adequately model the experimental equilibrium data. This signifies, according to a Scatchard plot, that these materials are homogeneous. Figure 8a and b compare the affinity spectra obtained from the continuous distribution model (Toth, as in Eq 14) and the discrete model. If the material is homogeneous, then the affinity spectra from the continuous distribution model and Toth isotherm produced similar results. The maximum binding affinity observed in the spectra calculated using the Toth isotherm is almost equal to the affinity parameter obtained from the discrete model. For the case of polymers 1 and 2, which are heterogeneous, the concentration of adsorption sites with low binding affinity deviates substantially from the ones obtained from the Toth isotherm. This can be expected if the adsorbent contains a relatively larger number of adsorption sites with lower affinity. The number of adsorption sites with high binding affinity matches with the ones obtained from the discrete models. Another notable feature in Fig 8 is that the binding affinity of the heterogeneous polymers ( 1 and 2 ) and their numbers obtained using discrete models differ from each other and this makes it difficult to compare the properties of the materials. The continuous distribution models can allow the material to be compared for their binding site properties as the affinity spectra of different materials can be generated within the same analytical window. For example, polymer 1 and 2 can be easily compared, as they are generated within the limits $K=0.12 \mathrm{mM}^{-1}$ to $385 \mathrm{mM}^{-1}$. Although these materials are structurally similar, polymer 1 contains a slightly higher number of binding sites with low binding affinity and a slightly less number of binding sites with higher binding affinity than polymer 2 . 

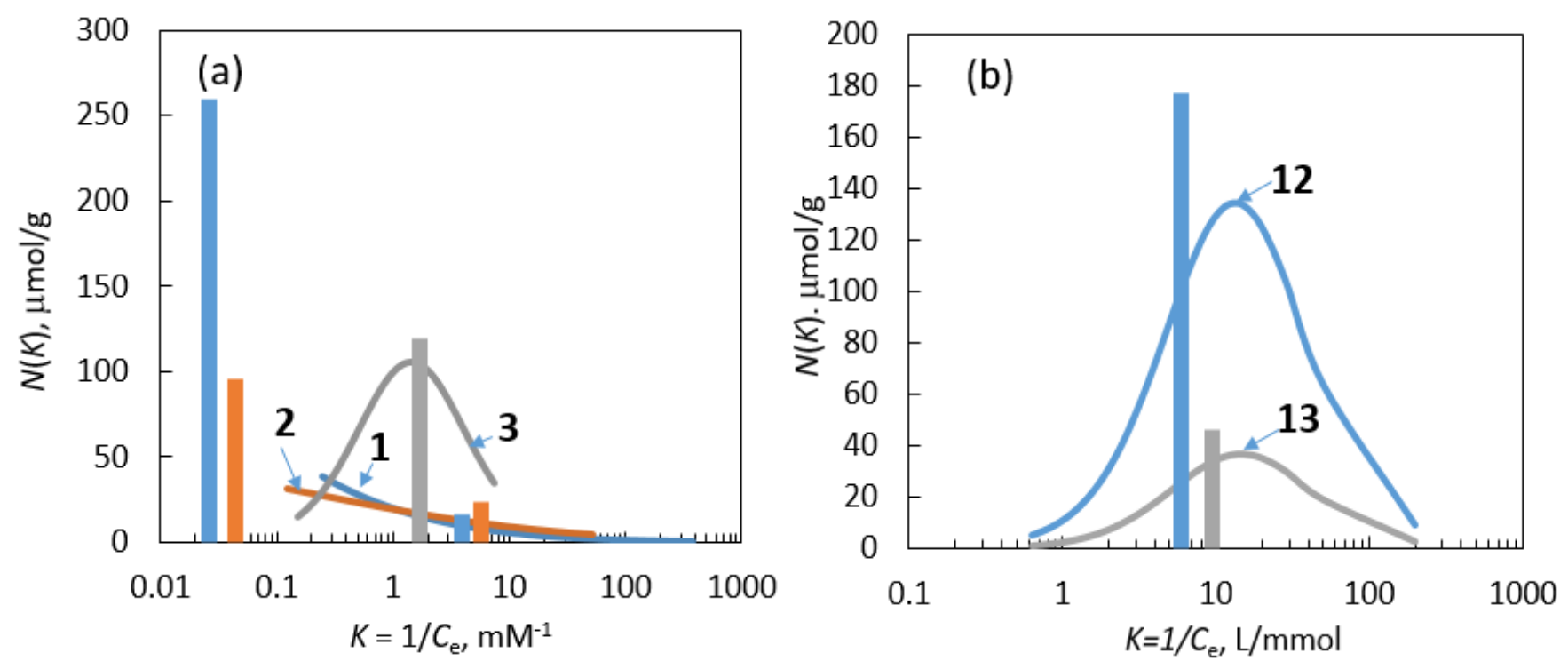

Fig. 8 (a) Affinity spectra of polymers 1-3 and sinapic recongnizing polymers 12 and 13 obtained using the Toth isotherm (solid lines) and a Scatchard plot method (bars).; (b) affinity spectra of sinapic acid-recognizing MIPs 12 and 13 obtained using the Toth isotherm and using the Scatchard plot method. Solid lines correspond to affinity spectra obtained using the Toth isotherm and the bars show the affinity spectra obtained using the Scatchard plot method.

The affinity spectrum can be generated using the Toth isotherm from its isotherm parameters without involving any initial guess on the number or type of adsorption sites. It is also noteworthy that non-linear regression analysis is a better approach than the commonly used linear regression analysis to calculate the affinity parameters in the discrete model.

\subsection{Binding affinity spectra of an adsorbent for different target molecules}

Adsorption isotherm parameters are sensitive to the properties of adsorbent, adsorbate and the experimental conditions such as temperature and pressure or concentration. This fact can be exploited to characterise the adsorbent for their binding properties to different target molecules. For instance, MIPs are developed using multiple templates to selectively remove multiple compounds that belong to different classes of compounds from the samples that are commonly encountered in the environment, e.g. for wastewater that contains more than one compounds. ${ }^{65-67}$ Polymer synthesis using multiple templates might produce a single material that can recognize multiple solutes and in some cases the material might contain larger cavities that can penalise the molecular recognition capability of the polymers. To test this, the Toth isotherm was used to characterise the molecular recognition capability of a polymer (14) for a mixture of pharmaceutical compounds ibuprofen, naproxen, ketoprofen, diclofenac and clofibric acid. The multi-targeting polymer was originally synthesised by Dai et al. to remove all of these pharmaceutical ingredients from contaminated wastewater. ${ }^{67} \mathrm{We}$ plot the affinity spectra obtained using eq (14) of polymer 14 for all these five pharmaceutical ingredients in Figure 9. The polymer exhibits molecular recognition capability towards all the five pharmaceutical ingredients. In terms of heterogeneity, the binding affinity spectra show that the materials contain different types of binding sites with different levels of selective affinity towards the five template compounds. Specifically, the unimodal and narrow affinity distribution of polymer 14 for clofibric acid suggests this material has a remarkable selective affinity towards this molecule, despite being a multiple templated polymer. For the other four pharmaceutical compounds, the binding affinity spectra seems to be broader and the use of multiple templates introduced heterogeneity in the binding sites or binding pockets. This can be expected as the multiple templates can form aggregates or arrange randomly within the polymer matrix and create binding pockets with shapes that are not the exact structural replica of the template molecules. Polymer 14 contains a heterogeneous distribution of binding sites for ibuprofen. For convenience, in Figure 9 we also showed the Toth isotherm parameter, $t$ for each of the solutes. Theoretically, the $t$ value ranges from 0 to 1 and the width of the affinity spectra increases with decrease in $t$ values. A value of $t=1$, indicates the material is 
homogeneous and contains only one type of binding sites. The value of $t$ if lesser than 1 , then material is heterogeneous and lower the value of $t$, higher is the material heterogeneity. Thus, according to the $t$ values and the width of the affinity spectra, the heterogeneity of polymer 14 for different target compounds in the decreasing order is given by: ibuprofen $>$ diclofenac $>$ ketoprofen > clofibric acid > naproxen. It is clear that polymer 14 has good applicability to selectively target or recognize naproxen. Additionally, the imprinting effect of naproxen was not influenced by the presence of other four molecular templates. In fact, the value of $t=1$ signifies that the polymer is homogeneous for this particular analyte (naproxen). The imprinting effect of ibuprofen was significantly diminished due to the multiple-templates used during the imprinting process as evidenced by the broadened affinity spectra. It is worth noting that the affinity parameter, $K$ in Figure 9 is deliberately expressed in molar units. Although, it is possible to plot the affinity in terms of gravimetric units, if the objective is to compare the affinity distribution of a single adsorbent for multiple solutes, it is useful to express the affinity parameter in molar units. In this way, it is easier compare the number of adsorption sites available under each type of adsorption sites as a function of different solutes that differ by molecular weight.

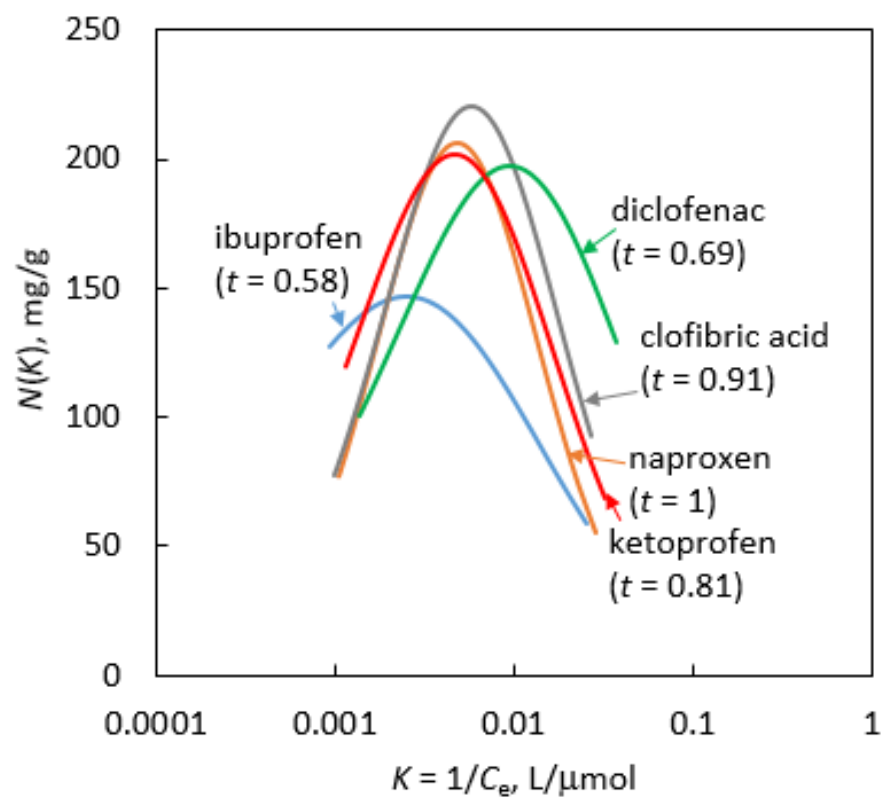

Fig. 9 Affinity spectra of multi-templated polymer (polymer 14) for ibuprofen, diclofenac, clofibric acid, naproxen and ketoprofen.

In this section, we only emphasised the usefulness of Toth isotherm to characterise an adsorbent that is specifically designed to target multiple solutes using MIPs as a case study. Though this idea can be generalised to characterise porous materials like MOFs, carbon allotropes, zeolites for their affinity towards different small molecules like $\mathrm{CO}_{2}, \mathrm{CH}_{4}, \mathrm{~N}_{2}$ and $\mathrm{H}_{2}$ within the same analytical window. It should be noted that the isotherm parameters are also sensitive to the experimental conditions including temperature. The shift in the affinity properties of the adsorbents due to temperature can be understood. Currently, the adsorptive performance of a single adsorbent for different target molecules is analysed from the isosteric heat. To generate an isosteric heat plot, an adsorption equilibrium experiments at (at least) two different temperatures must be performed. Alternatively, as shown in Figure 9, affinity spectra can be easily obtained from adsorption isotherm obtained at only temperature. Materials can be screened based on the width, the mean binding affinity and their concentration, $\mathrm{N}(\mathrm{K})$, especially when the adsorption equilibrium data is available at only one temperature.

\subsection{Comparison of Toth model with other binding models}

Theoretically, it is known that Toth isotherms can represent the experimental adsorption equilibrium data at both the subsaturation and saturation regions. Thus, the Toth isotherm can model the binding properties over the entire isotherm. Most of the adsorbents are heterogeneous and thus their binding properties cannot be simply modelled using the Langmuir Page | 22 
isotherm. In the literature, the Freundlich isotherm is commonly used to model the adsorption in the sub-saturation regions and to characterise the binding properties of the heterogeneous adsorbents. However, Freundlich assumes an exponential distribution of adsorption sites and thus cannot capture the actual binding properties at saturation conditions or in general higher concentrations. Another commonly-used model to deal with both homogeneous and heterogeneous adsorbents is the Langmuir-Freundlich expression. Both the Freundlich and Langmuir-Freundlich expressions suffer from the common limitation that both of these models cannot capture the lower end (Henry's region) of the adsorption isotherm. In such cases, a Toth can more accurately capture the adsorption than the Langmuir-Freundlich in the subsaturation and saturation regions of the adsorption isotherm. In many cases, adsorption experiments are performed only at lower solute concentrations or require the removal of trace quantities of pollutants from the bulk phase. One example is the capture of $\mathrm{CO}_{2}$ from flue gas where the target of the adsorbents is to remove $\mathrm{CO}_{2}$ at very low partial pressures $(\sim 0.1 \mathrm{bar}){ }^{68}$ At such low pressures, the adsorbent surface may not be completely filled with the $\mathrm{CO}_{2}$ molecules and it is more likely that many of the adsorption sites with low binding affinity remain unoccupied. Another example is $\mathrm{H}_{2}$ storage at room temperature. At $298 \mathrm{~K}$, the thermal motion of $\mathrm{H}_{2}$ molecules is high and the molecules' interactions with the adsorbent surfaces are essentially repulsive and weak. ${ }^{69}$ Thus, only at pressures closer to $\mathrm{H}_{2}$ storage conditions will the adsorption sites with high binding energy be sampled. Consequently, the adsorption isotherm will typically be of an exponential form and follow a Freundlich isotherm. In the case of MIPs, most of the MIPs are operated at very low concentration of adsorbate and thus only the adsorption sites with higher binding energy will be sampled during adsorption.

To demonstrate these facts, Figure 10a shows the affinity spectra of polymer 14 for naproxen and ibuprofen. The affinity spectra of two porous carbons for $\mathrm{CO}_{2}$ adsorption at $298 \mathrm{~K}$ are also calculated. The affinity spectra, calculated using the Toth isotherm, was compared with the affinity spectra obtained using Langmuir-Freundlich and Freundlich models. Th Freundlich and the Langmuir-Freundlich expressions used for comparison was taken from the literature. ${ }^{29,70}$ The Freundlich and Langmuir-Freundlich expressions and their binding affinity expressions are given in Table 3 of the supplementary file. The calculated adsorption isotherm parameters are given in Table 4 of the supplementary file. Both of the Toth expressions and Langmuir-Freundlich isotherm predict a narrow and unimodal distribution of binding energies for the case of naproxen. For the case of ibuprofen, the distribution is broad and unimodal in the saturation region followed by an exponentially decaying distribution in the subsaturation region. The Langmuir-Freundlich isotherm predicted a similar binding behaviour, the only difference is that the number of adsorption sites calculated using Toth isotherm is significantly higher than the ones obtained using the Langmuir-Freundlich isotherm. The peak maximum obtained using the Toth isotherm also deviates slightly from the peak maximum observed in affinity spectra obtained using the Langmuir-Freundlich isotherm. The Freundlich isotherm predicted an unrealistically exponentially decaying distribution for both naproxen and ibuprofen. The exponential trend reflects the theoretical background of the Freundlich isotherm rather than the property of the material. The Freundlich isotherm is applicable only if the equilibrium data are obtained in the subsaturation region. Irrespective of the nature of the adsorbent or the type of adsorption sites being sampled, Freundlich expression will produce an exponentially decaying trend. If the adsorption isotherms are obtained only in the sub-saturation region (for the case of $\mathrm{CO}_{2}$ adsorption at $298 \mathrm{~K}$; see Figure 2 of the supplementary file), then both Toth, Langmuir-Freundlich and Freundlich isotherm all predict an exponential distribution of binding energies. The magnitude of the number of adsorption sites varies depending on the models used. This is expected due to the different mathematical structure of these expressions. In general, the accuracy of the affinity spectra depends on how accurate the theoretical isotherm represents the experimental data. The best way to characterise the materials for the binding energies is to identify the best-fit isotherm and the binding model that correspond to best-fit isotherm can be used to obtain the affinity spectra. The results in Figure 10 clearly shows that the Toth isotherm can precisely capture the distribution of both high and low affinity binding sites, depending on the equilibrium data covered in the adsorption isotherm. 

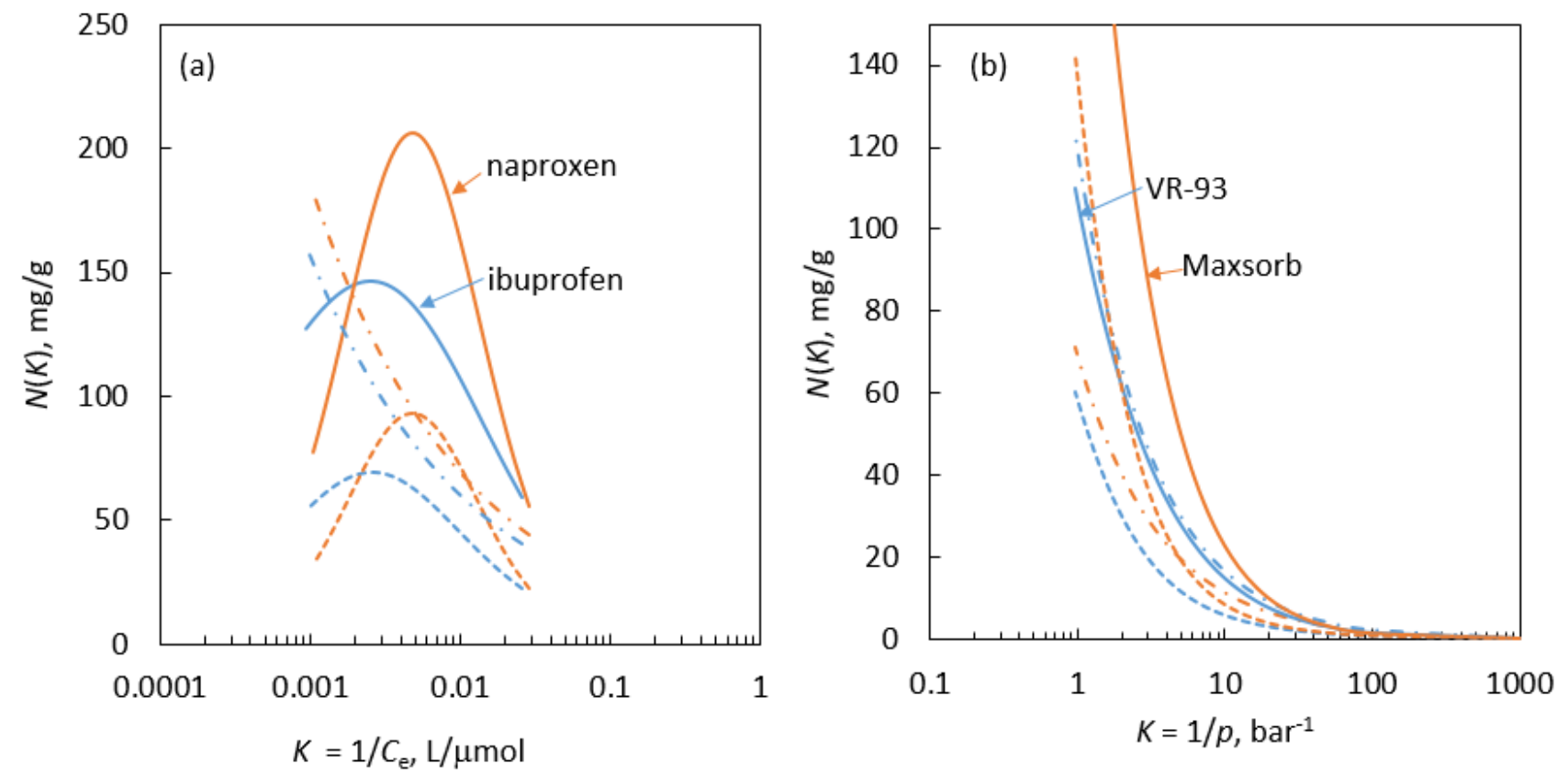

Fig. 10 (a) Affinity spectra of polymer 14 for ibuprofen and naproxen and (b) affinity spectra of two porous carbons for $\mathrm{CO}_{2}$ adsorption (solid lines: $N(K)$ obtained using Toth isotherm, dashed lines: $N(K)$ obtained using Langmuir-Freundlich isotherm, dashed and dotted lines: $N(K)$ obtained Freundlich isotherm).

The Toth isotherm contains three parameters and this means it can describe most of the experimental data better than the two-parameter models like Freundlich and Langmuir. In fact, in the field of gas adsorption, the Toth isotherm is one of the most widely used models for adsorption equilibrium modelling and design calculations. Valenzuela and Myers showed that this isotherm can accurately predict the experimental equilibrium data of several hundreds of such systems. ${ }^{71}$ This means their adsorbent properties can be easily estimated using the models proposed in this work. Another key advantage of the proposed model is that they can model both homogeneous and heterogeneous adsorbents. As stated earlier, the Toth isotherm also offers several advantages over the multi-site Langmuir isotherm. Depending on the number of types of adsorption sites assumed, the isotherm parameters to be determined increases when using a discrete model. The Scatchard plot will also produce binding affinity values that may differ from adsorbent to adsorbent; thus, the affinity spectra of different adsorbents cannot be easily compared based on the affinity parameters. Even though the Toth isotherm contains only three parameters, it can precisely capture the entire affinity distribution. Irrespective of the nature of the distribution being exponential or unimodal, the affinity spectra of different adsorbents can be placed within the same analytical window and their affinity distribution spectra can be compared. This is a desirable characteristic feature of the Toth isotherm and can become useful while comparing different classes of adsorbents.

\section{Conclusions}

Two affinity distribution functions are proposed based on the two different forms of the Toth isotherm. The affinity distribution functions can model both homogeneous and heterogeneous adsorption behaviour. These functions can generate affinity spectra within the same analytical windows, allowing characterisation of different classes of adsorbents. The method is straight-forward and simple and does not require any complex computations. The affinity spectra can be easily generated just from the Toth parameters and the isotherm parameters can be obtained using a simple non-linear regression analysis technique. Using the Toth isotherm, it is possible to generate the affinity spectra of several important materials, including molecularly imprinted polymers, metal-organic frameworks, highly porous and high surface area carbons and silica column. These examples are carefully chosen to demonstrate how the affinity spectra can be used to compare the performance of adsorbents, material quality, adsorbent design, and even to resolve an experimental paradox. 
In many industrial applications, the adsorption capacity of a material is often plagued by the presence of foreign compounds or impurities. The presence of any background impurities will affect the adsorption by directly competing with the target compounds and can sometimes irreversibly adsorb onto binding sites thus altering the adsorbent properties. In many situations, it is possible to deal with more than one pollutants from the bulk solution. In such cases, it is possible to characterise the adsorption mechanism in terms of which types of binding sites are vulnerable to the competing compounds using the affinity distribution. Affinity spectra can expose what type of binding sites are affected by the impurities. During adsorption of multicomponent solutes from a bulk solution, the selectivity of the adsorbent depends on the binding affinity of the individual components and what type of sites these components compete with each other. Affinity spectra can be used to bring information about which types of adsorption sites less favour the competition and this information can be used for material in order to improve the selectivity of the adsorbents.

To summarize, in this work, we calculated the affinity spectra for materials from different classes of adsorbents. The Toth isotherm can be taken as a universal model and can be used to characterise the binding behaviour other class of porous materials which are not considered in this work like zeolitic imidazolate frameworks (ZIFs), zeolites, covalent organic frameworks (COFs), carbon allotropes like carbon nanotube, porous graphene, polymers with intrinsic microporosity (PIMs) or any other new class of porous material.

\section{REFERENCES}

1 A. E. Creamer and B. Gao, Environ. Sci. Technol., 2016, 50, 7276-7289.

G. Srinivas, V. Krungleviciute, Z. X. Guo and T. Yildirim, Energy Environ. Sci., 2014, 7, 335-342.

3 K. V. Kumar, S. Gadipelli, K. Preuss, H. Porwal, T. Zhao, Z. X. Guo and M.-M. Titirici, ChemSusChem, 2017, 10, 199-209.

4 K. V. Kumar, K. Preuss, M. M. Titirici and F. Rodríguez-Reinoso, Chem. Rev., 2017, 117, 1796-1825.

5 S. Gadipelli, C. A. Howard, J. Guo, N. T. Skipper, H. Zhang, P. R. Shearing and D. J. L. Brett, Adv. Energy Mater., 2020, 10, 1903649.

$6 \quad$ L. Schlapbach and A. Züttel, Nature, 2001, 414, 353-358.

7 S. Wang, H. Nam, T. B. Gebreegziabher and H. Nam, Eng. Reports, , DOI:10.1002/eng2.12083.

8 F. V. S. Lopes, C. A. Grande, A. M. Ribeiro, J. M. Loureiro, O. Evaggelos, V. Nikolakis and A. E. Rodrigues, Sep. Sci. Technol., 2009, 44, 1045-1073.

9 L. H. de Oliveira, J. G. Meneguin, M. V. Pereira, J. F. do Nascimento and P. A. Arroyo, Chem. Eng. Commun., 2019, 206, 1544-1564.

10 T. Islamoglu, Z. Chen, M. C. Wasson, C. T. Buru, K. O. Kirlikovali, U. Afrin, M. R. Mian and O. K. Farha, Chem. Rev., , DOI:10.1021/acs.chemrev.9b00828.

11 L. H. Xie, X. M. Liu, T. He and J. R. Li, Chem, 2018, 4, 1911-1927.

12 K. Vellingiri, J. E. Szulejko, P. Kumar, E. E. Kwon, K. H. Kim, A. Deep, D. W. Boukhvalov and R. J. C. Brown, Sci. Rep., 2016, 6, 1-11.

13 W. K. Pui, R. Yusoff and M. K. Aroua, Rev. Chem. Eng., 2019, 35, 649-668.

14 M. Wen, G. Li, H. Liu, J. Chen, T. An and H. Yamashita, Environ. Sci. Nano, 2019, 6, 1006-1025.

T. Han, A. Nag, S. Chandra Mukhopadhyay and Y. Xu, Sensors Actuators, A Phys., 2019, 291, 107-143.

M. Woellner, S. Hausdorf, N. Klein, P. Mueller, M. W. Smith and S. Kaskel, Adv. Mater., 2018, 30, 1704679.

L. Wang, Sensors Actuators, A Phys., 2020, 307, 111984.

Y. Li, A. S. Xiao, B. Zou, H. X. Zhang, K. Le Yan and Y. Lin, Polyhedron, 2018, 154, 83-97.

I. Ali, A. A. Basheer, X. Y. Mbianda, A. Burakov, E. Galunin, I. Burakova, E. Mkrtchyan, A. Tkachev and V.

Grachev, Environ. Int., 2019, 127, 160-180.

20 J. QU, J. Environ. Sci., 2008, 20, 1-13.

M. Karnib, A. Kabbani, H. Holail and Z. Olama, in Energy Procedia, Elsevier Ltd, 2014, vol. 50, pp. 113-120.

M. Kikuchi, N. Tsuru and S. Shiratori, Sci. Technol. Adv. Mater., 2006, 7, 156-161.

Y. Zhang, J. Zhang and Q. Liu, Sensors (Switzerland), 2017, 17.

Page | 25 
K. Mosbach and O. Ramström, Bio/Technology, 1996, 14, 163-170.

K. V. Kumar, S. Gadipelli, B. Wood, K. A. Ramisetty, A. A. Stewart, C. A. Howard, D. J. L. Brett and F. Rodriguez-Reinoso, J. Mater. Chem. A, 2019, 7, 10104-10137.

I. M. Klotz and D. L. Hunston, Biochemistry, 1971, 10, 3065-3069.

R. J. Umpleby, S. C. Baxter, M. Bode, J. K. Berch, R. N. Shah and K. D. Shimizu, Anal. Chim. Acta, 2001, 435, $35-42$.

D. L. Hunston, Anal. Biochem., 1975, 63, 99-109.

R. J. Umpleby, S. C. Baxter, Y. Chen, R. N. Shah and K. D. Shimizu, Anal. Chem., 2001, 73, 4584-4591.

R. J. Umpleby, S. C. Baxter, A. M. Rampey, G. T. Rushton, Y. Chen and K. D. Shimizu, J. Chromatogr. B Anal. Technol. Biomed. Life Sci., 2004, 804, 141-149.

31 M. Thommes, K. Kaneko, A. V. Neimark, J. P. Olivier, F. Rodriguez-Reinoso, J. Rouquerol and K. S. W. Sing, Pure Appl. Chem., 2015, 87, 1051-1069.

32 K. V. Kumar, M. C. Monteiro de Castro, M. Martinez-Escandell, M. Molina-Sabio and F. Rodriguez-Reinoso, ChemPhysChem, 2010, 11, 2555-2560.

33 K. Vasanth Kumar, M. Monteiro De Castro, M. Martinez-Escandell, M. Molina-Sabio and F. Rodriguez-Reinoso, $J$. Phys. Chem. C, 2010, 114, 13759-13765.

34 K. V. Kumar, M. C. M. de Castro, M. Martinez-Escandell, M. Molina-Sabio and F. Rodriguez-Reinoso, Chem. Eng. J., 2011, 168, 972-978.

35 G. Scatchard, Ann. N. Y. Acad. Sci., 1949, 51, 660-672.

H. G. Weder, J. Schildknecet, R. A. Lutz and P. Kesselrinu, Determination of Binding Parameters from Scatchard Plots Theoretical and Practical Considerations, 1974, vol. 42.

A. K. Thakur, P. J. Munson, D. L. Hunston and D. Rodbard, Anal. Biochem., 1980, 103, 240-254.

52 J. J. Belbruno, Chem. Rev., 2019, 119, 94-119.

53 P. Szabelski, K. Kaczmarski, A. Cavazzini, Y. B. Chen, B. Sellergren and G. Guiochon, J. Chromatogr. A, 2002, 964, 99-111.

54 L. Anfossi, S. Cavalera, F. Di Nardo, G. Spano, C. Giovannoli and C. Baggiani, Polymers (Basel)., 2020, $12,1178$. Society of Chemistry, 2020, vol. 11, pp. 895-906.

61 D. Alezi, Y. Belmabkhout, M. Suyetin, P. M. Bhatt, L. J. Weseliński, V. Solovyeva, K. Adil, I. Spanopoulos, P. N. Trikalitis, A. H. Emwas and M. Eddaoudi, J. Am. Chem. Soc., 2015, 137, 13308-13318. 
62 J. Jiang, H. Furukawa, Y. B. Zhang and O. M. Yaghi, J. Am. Chem. Soc., 2016, 138, 10244-10251.

63 Http://arpa-e.energy.gov, Advanced Research Projects Agency - Energy (ARPA-E) U.S. Department of Energy, Methane Oppurtuities for Vehicular Energy (MOVE), http://arpa-e.energy.gov/?q=arpa-e-programs/move.

64 F. Gritti and G. Guiochon, Anal. Chem., 2003, 75, 5726-5738.

65 W. Lu, J. Liu, J. Li, X. Wang, M. Lv, R. Cui and L. Chen, Analyst, 2019, 144, 1292-1302.

66 L. M. Madikizela and L. Chimuka, J. Pharm. Biomed. Anal., 2016, 128, 210-215.

67 C. M. Dai, J. Zhang, Y. L. Zhang, X. F. Zhou, Y. P. Duan and S. G. Liu, Chem. Eng. J., 2012, 211-212, $302-309$.

68 P. G. Boyd, A. Chidambaram, E. García-Díez, C. P. Ireland, T. D. Daff, R. Bounds, A. Gładysiak, P. Schouwink, S. M. Moosavi, M. M. Maroto-Valer, J. A. Reimer, J. A. R. Navarro, T. K. Woo, S. Garcia, K. C. Stylianou and B. Smit, Nature, 2019, 576, 253-256.

69 K. V. Kumar, E. A. Müller and F. Rodríguez-Reinoso, J. Phys. Chem. C, 2012, 116, 11820-11829.

70 R. J. Umpleby, S. C. Baxter, A. M. Rampey, G. T. Rushton, Y. Chen and K. D. Shimizu, J. Chromatogr. B Anal. Technol. Biomed. Life Sci., 2004, 804, 141-149.

71 D. Valenzuela and A. L. Myers, Adsorption Equilibrium Data Handbook, Prentice Hall advanced reference series, 1988. 